\title{
Antibiotic pretreatment alleviates liver transplant damage in mice and humans
}

\author{
Kojiro Nakamura, ${ }^{1}$ Shoichi Kageyama, ${ }^{1}$ Takahiro Ito, ${ }^{1}$ Hirofumi Hirao, ${ }^{1}$ Kentaro Kadono, ${ }^{1}$ Antony Aziz, ${ }^{1}$ Kenneth J. Dery, ${ }^{1}$ \\ Matthew J. Everly, ${ }^{2}$ Kojiro Taura, ${ }^{3}$ Shinji Uemoto, ${ }^{3}$ Douglas G. Farmer, ${ }^{1}$ Fady M. Kaldas, ${ }^{1}$ \\ Ronald W. Busuttil, ${ }^{1}$ and Jerzy W. Kupiec-Weglinski \\ 'Dumont-UCLA Transplantation Center, Department of Surgery, Division of Liver and Pancreas Transplantation, David Geffen School of Medicine at UCLA, Los Angeles, California, USA. ${ }^{2}$ Terasaki Research \\ Institute, Los Angeles, California, USA. 'Division of Hepato-Biliary-Pancreatic Surgery and Transplantation, Department of Surgery, Graduate School of Medicine, Kyoto University, Kyoto, Japan.
}

\begin{abstract}
Although modifications of gut microbiota with antibiotics (Abx) influence mouse skin and cardiac allografts, its role in orthotopic liver transplantation (OLT) remains unknown. We aimed to determine whether and how recipient Abx pretreatment may affect hepatic ischemia-reperfusion injury (IRI) and OLT outcomes. Mice (C57BL/6) with or without Abx treatment (10 days) were transplanted with allogeneic (BALB/c) cold-stored (18 hours) livers, followed by liver and blood sampling ( 6 hours). We divided 264 human OLT recipients on the basis of duration of pre-OLT Abx treatment into control (Abx-free/Abx <10 days; $n=108$ ) and Abx treatment (Abx $\geq 10$ days; $n=156$ ) groups; OLT biopsy (Bx) samples were collected 2 hours after OLT ( $n=52$ ). Abx in mice mitigated IRI-stressed OLT (IRI-OLT), decreased CCAAT/enhancer-binding protein homologous protein (CHOP) (endoplasmic reticulum [ER] stress), enhanced LC3B (autophagy), and inhibited inflammation, whereas it increased serum prostaglandin E2 (PGE2) and hepatic PGE2 receptor 4 (EP4) expression. PCE2 increased EP4, suppressed CHOP, and induced autophagosome formation in hepatocyte cultures in an EP4-dependent manner. An EP4 antagonist restored CHOP, suppressed LC3B, and recreated IRI-OLT. Remarkably, human recipients of Abx treatment plus OLT (Abx-OLT), despite severe pretransplantation clinical acuity, had higher EP4 and LC3B levels but lower CHOP levels, which coincided with improved hepatocellular function (serum aspartate aminotransferase/serum aspartate aminotransferase [sALT/sAST]) and a decreased incidence of early allograft dysfunction (EAD). Multivariate analysis identified "Abx-free/Abx $<10$ days" as a predictive factor of EAD. This study documents the benefits of Abx pretreatment in liver transplant recipients, identifies ER stress and autophagy regulation by the PCE2/EP4 axis as a homeostatic underpinning, and points to the microbiome as a therapeutic target in OLT.
\end{abstract}

\section{Introduction}

Orthotopic liver transplantation (OLT) has become the standard of care for patients with end-stage liver disease or hepatic malignancies (1). Ischemia-reperfusion injury (IRI), a leading cause of early allograft dysfunction (EAD) and failure, represents a major risk factor in the development of acute and chronic rejection and contributes to the shortage of donor organs (2). However, despite obvious clinical importance, the mechanisms that account for liver IRI are not well understood, and new strategies to improve outcomes and expand the donor pool are warranted.

The gut microbiome, the reservoir of more than 100 trillion commensal microorganisms that exceeds the number of cells in the human body by 10-fold (3), contributes to inflammatory bowel disease (4), cardiovascular disease (5), obesity (6), diabetes (7),

\section{Related Commentary: p. 3054}

Authorship note: $\mathrm{KN}, \mathrm{SK}, \mathrm{TI}$, and $\mathrm{HH}$ contributed equally to this work. Conflict of interest: The authors have declared that no conflict of interest exists. Copyright: @ 2019, American Society for Clinical Investigation. Submitted: January 19, 2019; Accepted: May 21, 2019; Published: July 22, 2019 Reference information: / Clin Invest. 2019;129(8):3420-3434. https://doi.org/10.1172/JCl127550. and Parkinson disease (8). Early exposure to germs was found to modify IR-triggered kidney damage and inflammation in mice (9). More recent studies have also identified the microbiota as an environmental factor influencing survival of mouse skin and cardiac allografts $(10,11)$, whereas relatively little is known about its role in $\operatorname{OLT}(12,13)$. Although 2 weeks of antibiotic (Abx) pretreatment of the "donor" influenced Kupffer cell function and ameliorated hepatic IRI in mouse OLT (14), the clinical relevance of this strategy in cadaveric OLT is questionable. Others have shown that a 7-day regimen of Abx pretreatment in the "recipient" only attenuated hepatic damage in rat OLT (15), but the underlying mechanisms remain unknown. As liver transplant patients routinely undergo prophylactic Abx treatment, whether prolonged Abx pretreatment is truly beneficial for OLT outcomes remains to be elucidated.

The endoplasmic reticulum (ER), the primary cellular organelle of protein synthesis, is highly sensitive to stress stimulation. Stressed ER is characterized by a reduced capacity to handle newly synthesized proteins, leading to the accumulation and aggregation of unfolded proteins in the ER lumen. As the principal organ of protein synthesis, liver ER stress involves various pathologies, including hepatic IRI (16-18). The CCAAT/enhancer-binding protein homologous protein (CHOP), a transcription factor and ER stress marker, plays a central role in ER stress-induced apoptosis (19- 
21), whereas CHOP-deficient mice were protected against warm IRI (22). On the other hand, Abx-mediated gut microbiota modifications in a diet-induced chronic liver inflammation mouse model decreased ER stress markers, including CHOP expression (23). However, it remains unknown whether and how Abx may regulate ER stress in OLT. Autophagy is an evolutionarily conserved intracellular self-digesting pathway responsible for maintaining energy homeostasis and removing long-lived or damaged organelles and proteins (24). A growing body of evidence indicates a tissueprotective function of autophagy in various pathologic states such as aging, diabetes, and neurodegenerative diseases (25), as well as in liver IRI (26-29). Meanwhile, the influence of the microbiome on hepatic autophagy in OLT is yet to be determined.

Prostaglandin E2 (PGE2) is a bioactive lipid that elicits a wide range of biological functions through 4 distinct $\mathrm{G}$ protein-coupled receptor subsets: prostaglandin E2 receptors 1-4 (EP1-EP4) (30). Previous studies have demonstrated a cytoprotective function of extrinsic PGE2 in preclinical liver injury models $(31,32)$. Although EP4 has been identified as a key binding partner in protection against warm liver IRI (33), the role of the PGE2/EP4 axis in hepatocyte ER stress and autophagy regulation in OLT has not been studied to date. On the other hand, the expression of COX2, a responsive enzyme for PGE2 production, is constitutively marginal in livers as compared with COX2-proficient organs such as cerebral cortex or the gastrointestinal tract (34). Indeed, Abx-mediated microbiota alterations may increase gut-derived systemic PGE2 levels and contribute to disease physiology in remote organs (35). Hence, we asked whether and how gut-derived PGE2 may be influenced by recipient microbiota after Abx treatment in OLT recipients.

This study documents the benefits of recipient Abx pretreatment and identifies a molecular crosstalk between gut-derived PGE2 and its response via hepatic EP4 signaling in IR-stressed OLT. In the experimental arm, we used a clinically relevant allogeneic mouse OLT model to show that recipient Abx pretreatment increased serum PGE2 levels, while decreasing ER stress, enhancing the autophagy pathway, and attenuating liver IRI in an EP4-dependent manner. In the clinical arm, liver transplant patients subjected to a prolonged Abx regimen prior to surgery had increased EP4 and lower CHOP levels, but higher LC3B levels, as well as improved hepatocellular function. Remarkably, despite parameters of severe clinical acuity before transplantation, our data identify extended Abx pretreatment as an independent predictor for averting EAD in OLT patients.

\section{Results}

Recipient Abx pretreatment attenuates, but adjunctive fecal microbiota transfer recreates, hepatic IRI in mouse allogeneic OLT. We first aimed to determine the influence of Abx treatment on IRI severity in a clinically relevant allogeneic mouse OLT model (BALB/c> $\mathrm{C} 57 \mathrm{BL} / 6)$ with ex vivo cold storage $\left(4^{\circ} \mathrm{C}\right.$ for 18 hours), which mimics marginal human liver grafts. Mouse OLT recipients pretreated for 10 days $(14,15)$ with oral Abx (amoxicillin, $50 \mathrm{mg} / \mathrm{mL}$ ) showed decreased serum aspartate aminotransferase (sAST) levels (OLT = $7047 \pm 1332$ vs. OLT $+\mathrm{Abx}=3609 \pm 447 \mathrm{IU} / \mathrm{L}, P=0.0317$; Figure $1 \mathrm{~A})$; attenuated sinusoidal congestion, edema/vacuolization and hepatocellular necrosis (Figure 1B); decreased Suzuki's histological grading of IRI $(\mathrm{OLT}=6.8 \pm 0.6$ vs. $\mathrm{OLT}+\mathrm{Abx}=3.6 \pm 0.5, P$
$=0.0003$; Figure 1C); and suppressed frequency of $\mathrm{TUNEL}^{+}$cells per high-power field $(\mathrm{HPF})(\mathrm{OLT}=64.0 \pm 8.6 \mathrm{vs}$. $\mathrm{OLT}+\mathrm{Abx}=23.4$ $\pm 3.0, P=0.0002$, Figure $1, \mathrm{~B}$ and $\mathrm{C}$ ) after 6 hours of reperfusion, as compared with control OLT mice. Thus, pretreatment of the recipient only for 10 days with Abx ameliorated liver IRI and alleviated hepatocellular death following OLT.

To determine whether gut microbiota composition may have influenced hepatocellular damage in IR-stressed OLT, we conducted a separate series of experiments in Abx-pretreated mice with or without adjunctive fecal microbiota transfer (FMT) (Supplemental Figure 1; supplemental material available online with this article; https://doi.org/10.1172/JCI127550DS1). Indeed, we found that FMT from untreated naive mice $(\mathrm{C} 57 \mathrm{BL} / 6)$ recreated hepatic IRI in Abx-pretreated, stress-resistant allo-OLT (C57BL/6), as evidenced by liver histology (Supplemental Figure 1A); a significantly higher frequency of $\mathrm{TUNEL}^{+}$cells $/ \mathrm{HPF}(\mathrm{OLT}+\mathrm{Abx}=15.2 \pm 2.2 \mathrm{vs}$. $\mathrm{OLT}+\mathrm{Abx}+\mathrm{FMT}=104.2 \pm 2.8, P<0.0001$; Supplemental Figure $1, \mathrm{~B}$ and $\mathrm{E})$; sAST levels (OLT $+\mathrm{Abx}=4274 \pm 615$ vs. OLT $+\mathrm{Abx}+$ FMT $=7485 \pm 379 \mathrm{IU} / \mathrm{L}, P=0.0062$; Supplemental Figure $1 \mathrm{C})$; and Suzuki's histological IRI grading (OLT $+\mathrm{Abx}=2.9 \pm 0.6$ vs. OLT $+\mathrm{Abx}+\mathrm{FMT}=6.5 \pm 0.3, P=0.0001$; Supplemental Figure 1D) These results further support the notion that the gut microbiome may dictate the severity of IRI-stressed OLT (IRI-OLT).

Abx treatment decreases the proinflammatory immune phenotype in IR-stressed mouse OLT. Since the release of danger-associated molecular patterns (DAMPs), such as high-mobility group box 1 (HMGB1), from injured cells is the key event triggering a cascade of inflammatory cytokines and chemokines, which further exacerbate organ damage, we next aimed to evaluate the impact of recipient Abx treatment on the release of HMGB1 and innate immune responses. Six hours after reperfusion, OLT recipients pretreated with Abx showed lower serum HMGB1 levels (Figure 1D); a decreased frequency of $\mathrm{CD}^{+} 8^{+}$(macrophages) and $\mathrm{Ly}^{6} \mathrm{G}^{+}$ (neutrophils) cells (Figure 1, E and F); lower serum MCP1 levels (Figure 1D); and suppressed levels of mRNA coding for CD68, Ly6G, MCP1, CXCL2, and CXCL10 (Figure 1G). Thus, preserved hepatocellular function after $\mathrm{Abx}$ therapy in recipient mouse (Figure 1, A-C) was accompanied by suppressed inflammation in IR-stressed OLT (Figure 1, D-G).

Abx treatment increases serum PGE2 while enhancing hepatic EP4 expression after murine OLT. Since PGE2 was shown to hamper proinflammatory responses in various immune cells $(36,37)$, we next focused on the Abx-PGE2 crosstalk in our experimental model system. Abx therapy enhanced the expression of COX2, a responsive enzyme for the production of PGE2 in the intestine (Figure 2, A and B). In naive mice, serum PGE2 metabolite (PGEM) levels were significantly higher in portal blood compared with levels in peripheral blood, whereas the Abx regimen further increased portal PGEM expression (Figure 2C). In parallel, Abx pretreatment enhanced PGEM levels in peripheral blood 6 hours after reperfusion in OLT as compared with levels in the control OLT group (Figure 2C). With four PGE2 receptors (EP1, EP2, EP3, and EP4) expressed in naive and ischemic livers, but only treatment with a selective EP4 agonist significantly reducing liver IRI (33), we focused here on PGE2/EP4 signaling. Indeed, addition of PGE2 increased mRNA (Figure 2D) and protein (Figure 2E) levels of EP4 in primary mouse hepatocyte cultures. Moreover, Abx ther- 
A

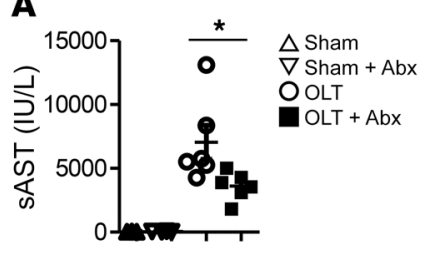

B

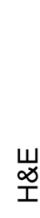

Sham

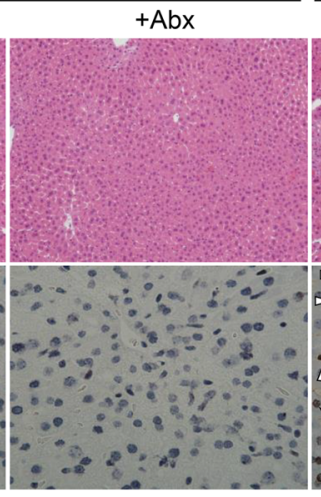

OLT

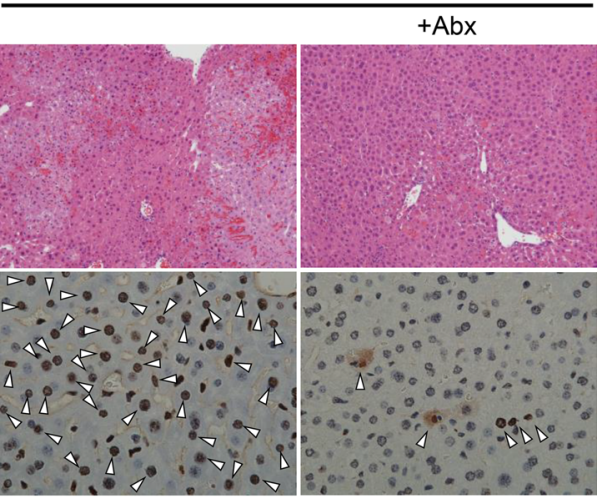

D
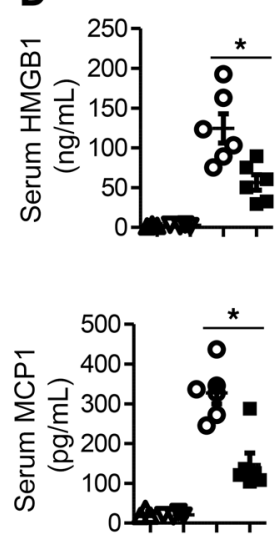

E
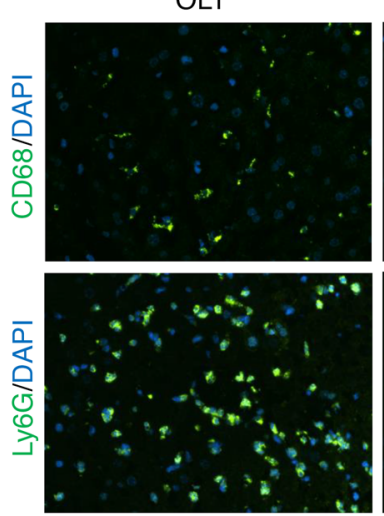

E
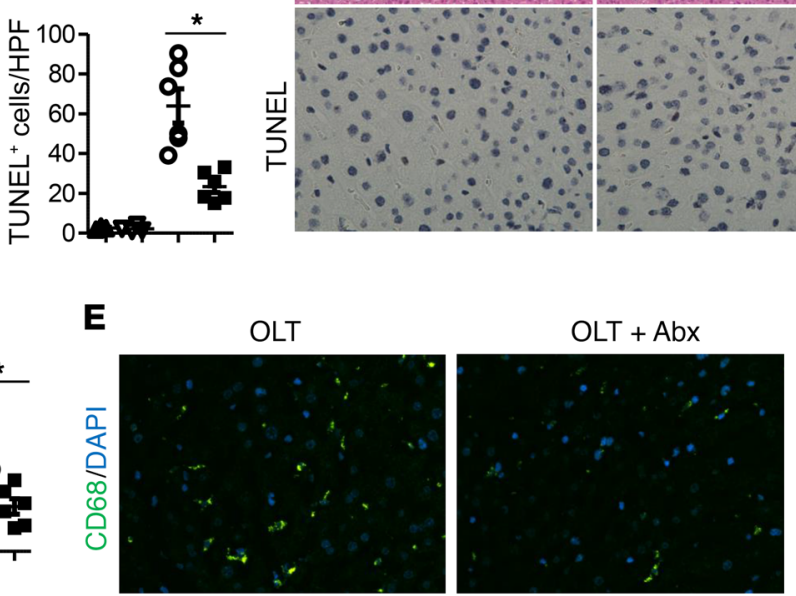

$\mathbf{F}$
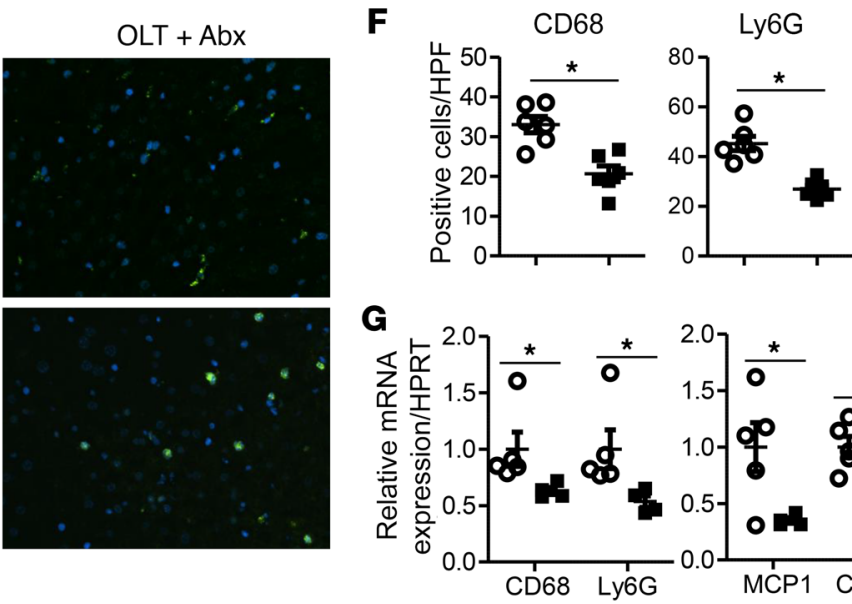

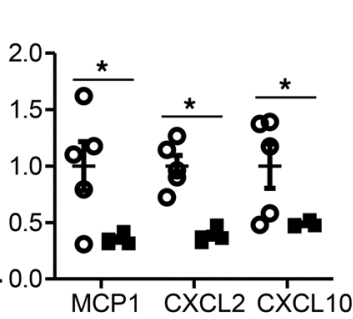

Figure 1. Recipient Abx pretreatment mitigates IRI, while suppressing inflammatory responses in mouse OLT. Mouse (BALB/C) livers subjected to 18 hours of cold storage were transplanted into allogeneic mice (C57BL/6). Some recipients were treated with Abx for 10 days before OLT (OLT + Abx). OLT and serum samples were analyzed 6 hours after reperfusion. The sham group underwent the same procedures except for OLT. (A) sAST levels were measured. (B) Representative H\&E staining (original magnification, $\times 100$ ) and TUNEL staining (original magnification, $\times 400$ ). Arrowheads indicate TUNEL ${ }^{+}$dead cells. (C) Suzuki's histological grading of liver IRI and quantification of TUNEL + cells/HPF. (D) Serum HMCB1 (ng/mL) and MCP1 (pg/mL) levels were measured by ELISA. (E) Representative immunohistochemical staining of hepatic CD68 ${ }^{+}$and Ly6G $\mathrm{C}^{+}$cells (original magnification, $\left.\times 400\right)$. (F) Quantification of hepatic $\mathrm{CD68}^{+}$and Ly6C ${ }^{+}$cells. (G) qRT-PCR detection of mRNA coding for CD68, Ly6C, MCP1, CXCL2, and CXCL10 in OLT. Data were normalized to HPRT gene expression. Data indicate the mean \pm SEM. ${ }^{*} P<0.05$ vs. OLT, by Student's $t$ test or 1-way ANOVA followed by Tukey's HSD test ( $\left.n=4-6 / g r o u p\right)$.

apy in recipients increased hepatic EP4 expression, as evidenced by Western blot and IHC analyses, with EP4 being expressed primarily by parenchymal cells in the periportal (zone 1) rather than the perivenous (zone 3) area (Figure 2, F and G). These data indicate that Abx pretreatment increases gut-derived PGE2 and hepatic EP4 expression.

PGE2 decreases CHOP, suppresses $m$ TORC1 activity, and enhances autophagy in mouse hepatocytes via EP4. We next aimed to determine the influence of PGE2 on hepatocyte ER stress and autophagy signaling pathways, with CHOP expression examined as an ER stress marker. As mTORC1 inhibition is essential to enhance the autophagy program (38), phosphorylated S6K (p-S6K), a marker of mTORC1 activity, was also evaluated (39). Addition of PGE2 to mouse primary hepatocyte cultures suppressed CHOP and p-S6K (Figure 3A) and enhanced LC3B aggregation, a marker of autophagosome formation for monitoring autophagy (Figure 3C). Moreover, addition of an EP4 antagonist (20 nM, ONO-AE3-208) restored $\mathrm{CHOP}$ and $\mathrm{p}-\mathrm{S} 6 \mathrm{~K}$ expression and inhibited LC3B aggregation in PGE2-treated hepatocytes (Figure 3, B and C). Thus,
PGE2 suppressed the ER stress response in hepatocytes, while enhancing autophagy in an EP4-dependent manner.

EP4 antagonism restores $C H O P$, inhibits autophagy, and recreates IRI in Abx-pretreated OLT recipient mice. Having demonstrated the induction of gut-derived PGE2 and hepatic EP4 signaling with recipient Abx pretreatment (Figure 2) as well as EP4-dependant ER stress and autophagy regulation by PGE2 in hepatocyte cultures (Figure 3, A-C), we asked whether the PGE2/EP4 axis may contribute to OLT protection after Abx therapy. Six hours after reperfusion, Abx-conditioned OLT showed decreased CHOP, inhibited p-S6K (mTORC1 activity), and increased the levels of LC3B-I plus LC3B-II and LC3B-II, as determined by Western blotting (Figure 3D), whereas OLT IHC analysis consistently revealed enhanced LC3B expression in parenchymal cells (Figure 3E). In addition, supplementation of an EP4 antagonist (ONO-AE3-208) to the Abx regimen recreated hepatic IRI-OLT, i.e., it deteriorated liver architecture (Figure 4A), augmented Suzuki's histological scores (Figure 4B), enhanced the frequency of TUNEL ${ }^{+}$cells (Figure 4, A and C), and increased sAST levels (Figure 4D), simultane- 


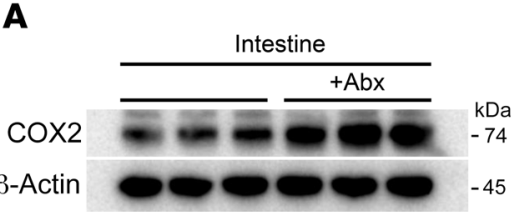

B
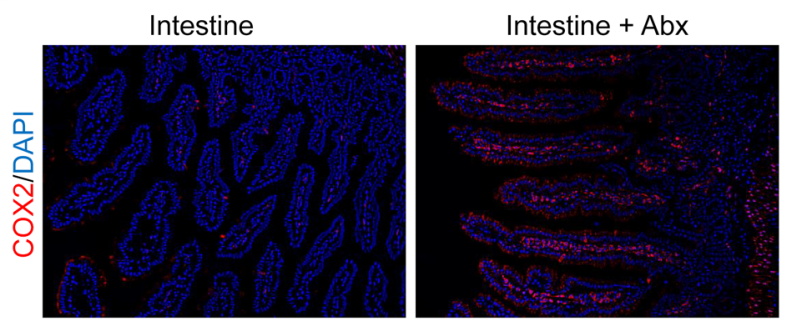

F
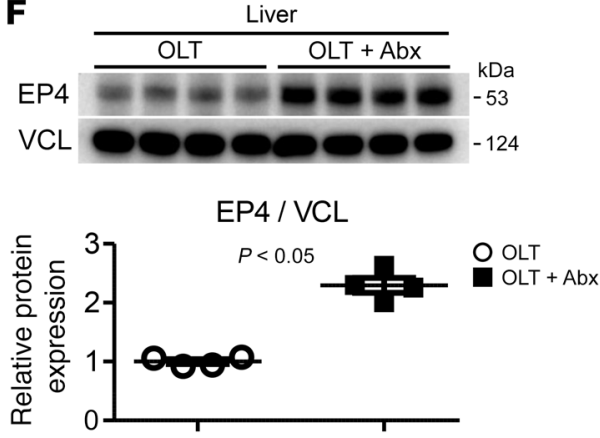

G
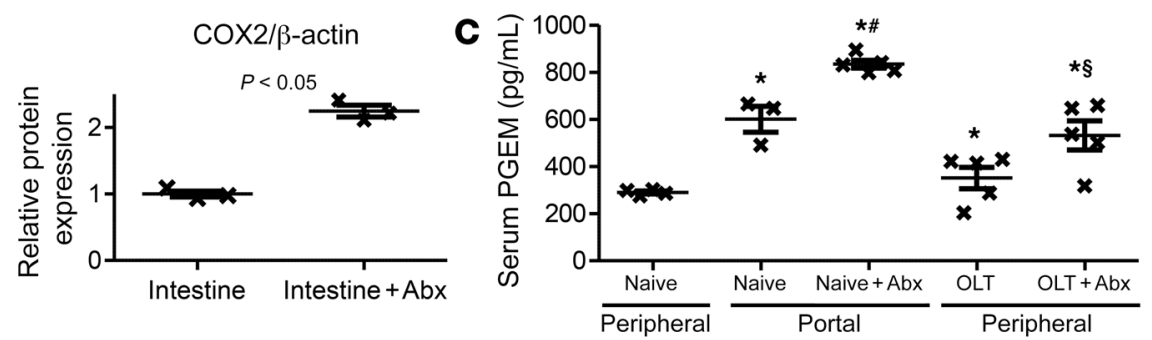

D

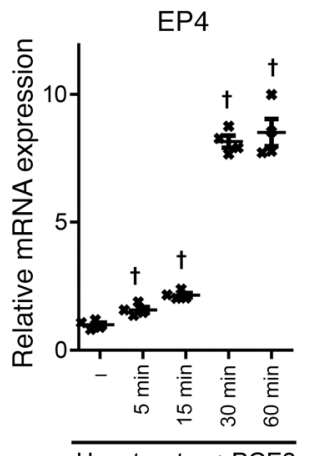

$\overline{\text { Hepatocytes + PGE2 }}$
E
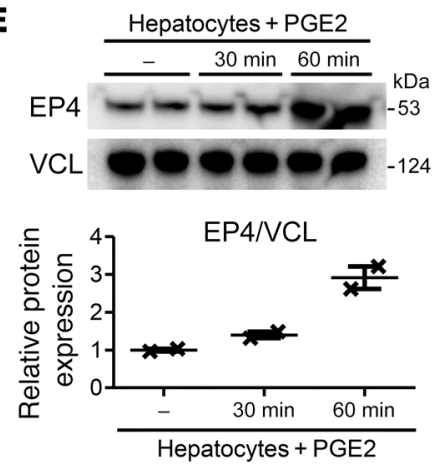

Liver

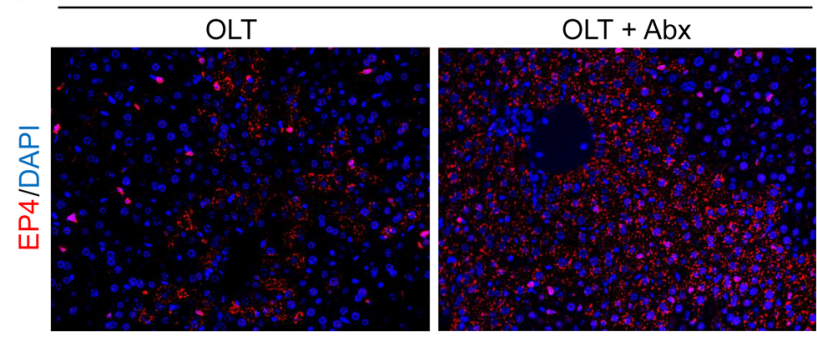

Figure 2. Abx treatment increases serum PGE2 and enhances EP4 expression in OLT. (A-C) Intestine tissue and serum samples from portal veins of naive and Abx-treated (10 days) C57BL/6 mice were analyzed. (C, F, and C) BALB/C livers subjected to 18 hours of cold storage were transplanted into C57BL/6 mice pretreated or not with $A b x$ for 10 days, followed by serum and liver sampling 6 hours after OLT. ( $\mathbf{D}$ and $\mathbf{E}$ ) Primary mouse hepatocytes were incubated with or without PGE2 (5 $\mu \mathrm{M}$ ) for the indicted time periods. (A) Western blot detection and the relative intensity ratio of COX2 in intestines of naive and Abx-treated mice. $\beta$-Actin expression served as an internal control and was used for normalization ( $n=3 / g r o u p)$. (B) Representative immunohistochemical staining of COX2 in intestines from naive and Abx-treated mice ( $n=3 /$ group). Original magnification, $\times 100$. (C) Serum PGEM levels were measured by ELISA ( $n=4$-6/group). ${ }^{*} P<0.05$ versus peripheral/naive; ${ }^{*} P<0.05$ versus portal/naive; ${ }^{\$} P<0.05$ versus peripheral/OLT. (D) qRT-PCR detection of mRNA coding for EP4 with TBP normalization ( $n=4$ /group). ${ }^{\dagger} P<0.05$ versus hepatocytes without PGE2. (E) Western blot detection and relative intensity ratios of EP4 in hepatocytes. VCL expression served as an internal control and was used for normalization ( $n=2 /$ group). (F) Western blot detection of EP4 and its relative intensity ratio with VCL normalization in OLT ( $n=4$ /group). (C) Representative immunohistochemical staining of EP4 in OLT with or without recipient Abx treatment $(n=3$ /group). Original magnification, $\times 200$. Data indicate the mean \pm SEM. Statistical significance was determined by Student's $t$ test or 1 -way ANOVA followed by Tukey's HSD test.

ously with restored $\mathrm{CHOP}$ and $\mathrm{p}-\mathrm{S} 6 \mathrm{~K}$ expression and suppressed expression of both LC3B-I plus LC3B-II and LC3B-II (Figure 4E). These results indicate that the PGE2/EP4 axis played a key role in ER stress and autophagy regulation and graft protection against IR stress following pretreatment of OLT recipients with Abx.

Human OLT recipients with extended Abx treatment exhibit higher levels of hepatic EP4 expression. Having demonstrated the importance of hepatic EP4 signaling for mouse OLT protection after 10 days of Abx treatment, we next aimed to evaluate whether adjunctive Abx therapy may promote EP4 signaling in liver transplant patients. Postreperfusion hepatic biopsy (Bx) (2 hours after portal reperfusion) from 52 human OLT patients (October 2013August 2015) were analyzed by Western blotting, with patients divided into control (recipients of pre-OLT Abx-free or pre-OLT Abx $<10$ days, $n=24$ ) and Abx treatment (recipients with pre-OLT Abx $\geq 10$ days, $n=28$ ) groups on the basis of the duration of preOLT Abx therapy, except for a routine preventive Abx regimen against surgical site infection (SSI) (Figure 5A). Clinical parameters for the recipients' and donors' OLT graft Bx samples $(n=52)$ are listed in Supplemental Tables 1 and 2. The Abx-treated patient cohort was younger $(P=0.008)$ and had a higher rate of concomitant hepatocellular carcinoma (HCC) $($ control $=66.7 \%$ vs. $\mathrm{Abx}=$ $14.3 \%, P<0.001)$, as well as a higher median model for end-stage liver disease $(\mathrm{MELD})$ score (control $=15 \mathrm{vs}$. $\mathrm{Abx}=37$, median, $P<$ 0.001), whereas there was no correlation between Abx grouping and donor/graft parameters. Western blot analyses revealed sig- 
A
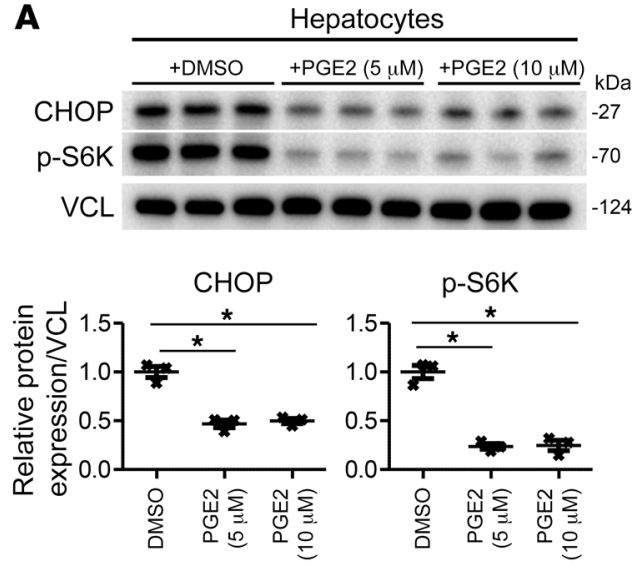

B
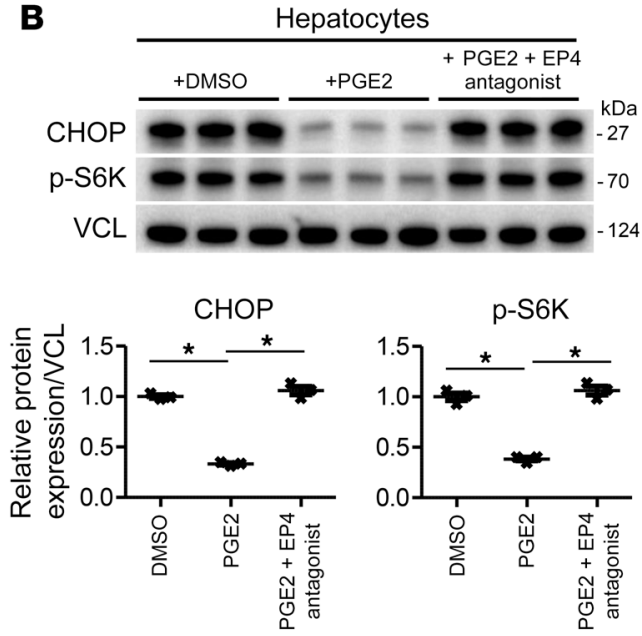

C

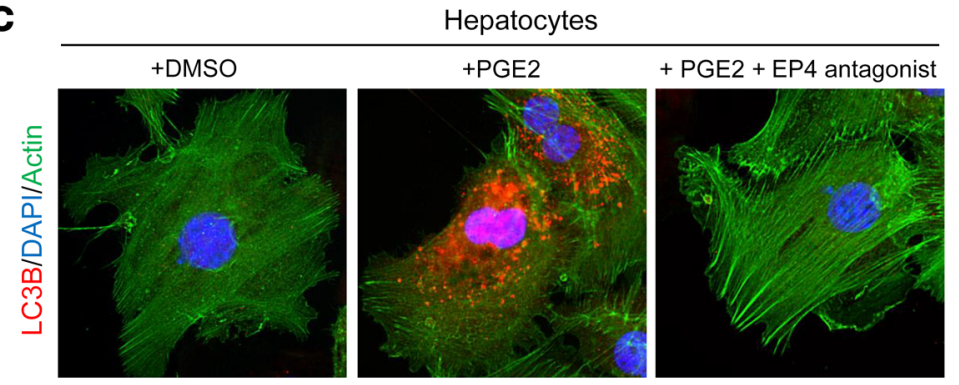

D
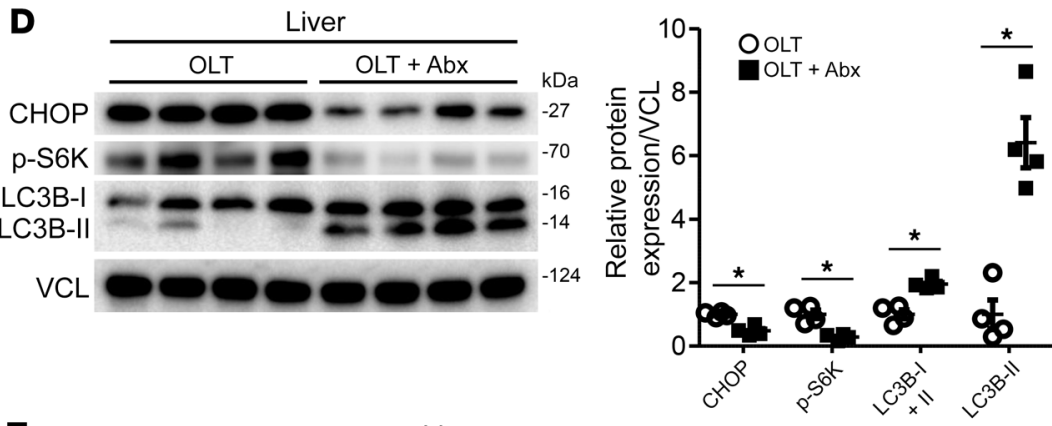

$\mathbf{E}$
Liver

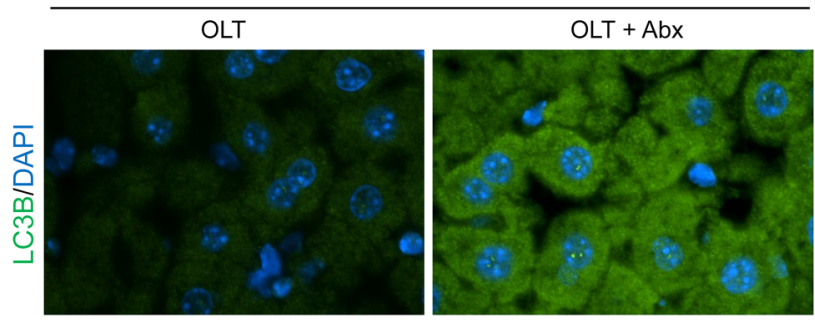

Figure 3. CHOP expression is inhibited concomitantly with LC3B enhancement via PGE2/EP4 signaling in hepatocytes as well as by recipient Abx pretreatment in OLT. (A) Primary mouse hepatocytes were incubated with DMSO, PGE2 (5 $\mu$ M), or PGE2 (10 $\mu$ M) for 60 minutes. Western blot detection and relative intensity ratios of CHOP and p-S6K. VCL expression served as an internal control and was used for normalization ( $n=3 / g r o u p)$. (B) Primary mouse hepatocytes were incubated with PGE2 $(5 \mu \mathrm{M})$ for 60 minutes, and the EP4 antagonist (20 nM, ONO-AE3-208) was supplemented 15 minutes prior to PGE2 incubation in some cell cultures. Western blot detection and relative intensity ratios of CHOP and p-S6K with VCL normalization ( $n=3 /$ group). (C) Representative immunohistochemical images show detection of LC3B (red) in hepatocytes cultured with DMSO, PGE2, or PGE2 plus the EP4 antagonist (actin: green; DAPI: blue). $n=3$. Original magnification, $\times 400$. (D) BALB/c mouse livers subjected to 18 hours of cold storage were transplanted orthotopically into allogeneic C57BL/6 mice pretreated or not with Abx for 10 days, followed by OLT sampling 6 hours after reperfusion. Western blot detection of CHOP, $\mathrm{p}-\mathrm{S} 6 \mathrm{~K}$, and LC3B and relative quantifications with VCL normalization in OLT ( $n=4 /$ group). (E) Representative immunohistochemical detection of LC3B (green) in OLT, with or without recipient Abx pretreatment (DAPI: blue). $n=3$. Original magnification, $x 400$. Data indicate the mean \pm SEM. ${ }^{*} P<0.05$, Student's $t$ test or 1-way ANOVA followed by Tukey's HSD test.

nificantly higher EP4 expression in OLT Bx samples from the Abx treatment group as compared with expression levels in samples from the control group $(P=0.0218$, Figure $5 \mathrm{~B})$. Representative images of EP4 staining in Bx samples are shown in Figure 5C.

$A b x$ pretreatment decreases $C H O P$ and p-S6K, enhances $L C 3 B$ expression, and mitigates IRI in human OLT. Recipients in the Abx treatment group had lower p-S6K $(P=0.0332)$ but increased LC3B-I plus LC3B-II $(P=0.0381)$ and LC3B-II $(P=0.0074)$ expression profiles, and $\mathrm{CHOP}$ expression after Abx therapy failed to reach statistical significance $(P=0.0563)$ between the 2 groups (Figure 6, A and B). Representative images of LC3B and TUNEL staining from Bx samples are shown in Figure 6C. Quantitative reverse transcription PCR (qRT-PCR) mRNA quantification of hepatic CD68 (macrophages) and cathepsin G (neutrophils) showed that the Abx-treated group trended toward decreased CD68 $(P=0.1522)$ and cathepsin $\mathrm{G}(P=0.1062)$ levels (Figure 6D). Despite higher MELD scores and comparable pretransplantation hepatocellular function (Supplemental Table 1), OLT recipients in the Abx treatment group had decreased liver enzyme levels (serum alanine aminotransferase [sALT]: $P=$ 0.0218 , sAST: $P=0.0474$; Figure $6 \mathrm{E}$ ) and trended toward a lower incidence of EAD compared with the control group (control = $25.0 \%$ vs. $\mathrm{Abx}=17.9 \%, P=0.3853)$. Hence, consistent with our mouse data, human liver transplant patients subjected to prolonged Abx conditioning prior to surgery had enhanced hepatic EP4 signaling, suppressed ER stress but augmented autophagy pathways, and mitigated IRI-OLT.

Extended Abx pretreatment decreases the incidence of $E A D$ in human clinical OLT. In addition to $52 \mathrm{Bx}$ samples (Figure 5A), OLT patients without Bx samples $(n=212)$ during the same recruitment 
A

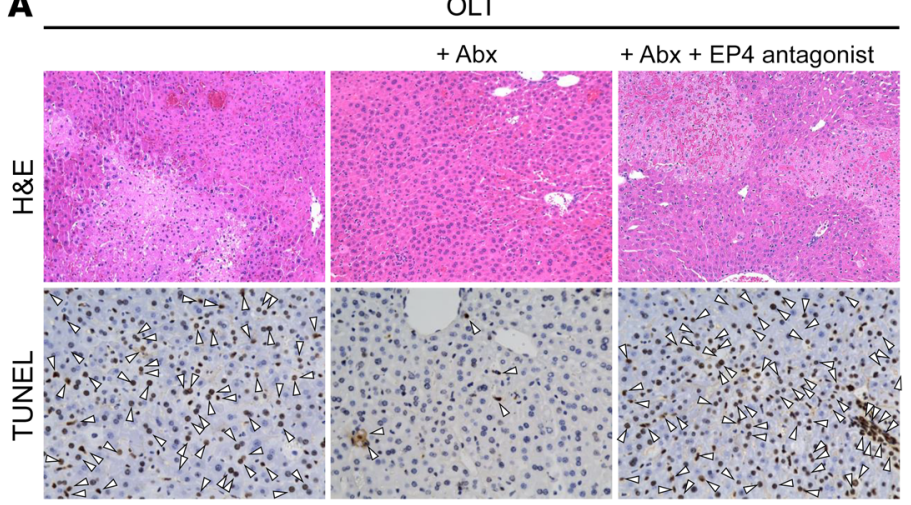

B

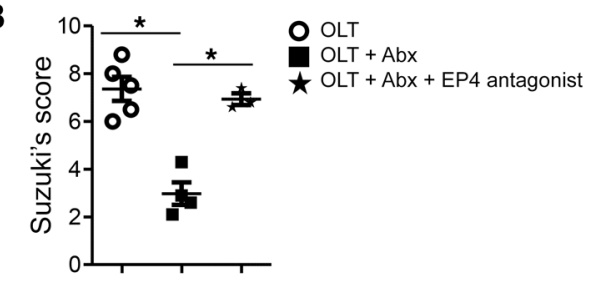

C

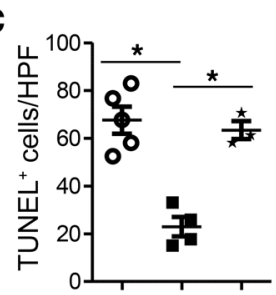

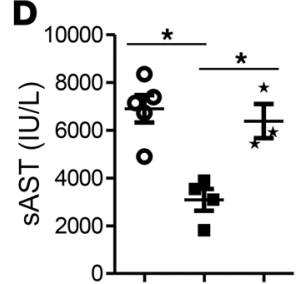
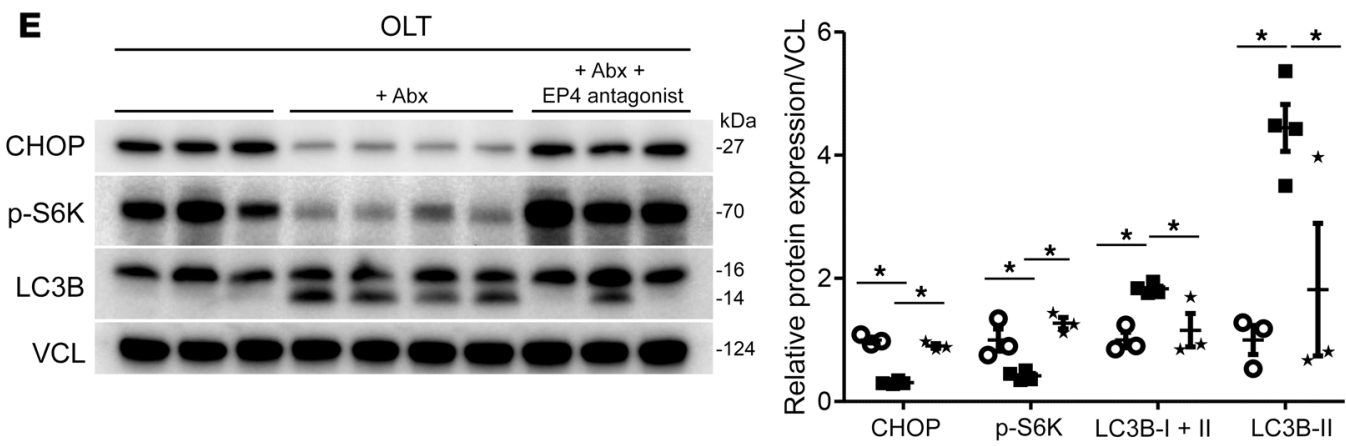

Figure 4. EP4 antagonism restores CHOP, decreases LC3B, and recreates IR-hepatocellular damage in Abx-pretreated OLT recipients. BALB/C livers subjected to 18 hours of cold storage were transplanted orthotopically into C57BL/6 mice, followed by serum and liver sampling 6 hours after OLT. Some OLT recipients were treated with Abx for 10 days before OLT, with or without an oral EP4 antagonist ( $5 \mathrm{mg} / \mathrm{kg}$, ONO-AE3-208 at 0 and 3 hours after reperfusion). (A) Representative H\&E staining (original magnification, $\times 100)$ and TUNEL staining (original magnification, $\times 200$ ). Arrowheads indicate TUNEL ${ }^{+}$ dead cells. (B) Suzuki's histological grading of liver IRI ( $n=3-5 /$ group). (C) Quantification of TUNEL cells/HPF ( $n=3-5 / g r o u p)$. (D) sAST levels were measured ( $n=3-5$ /group). (E) Western blot detection of CHOP, p-S6K, and LC3B and relative intensity ratios with VCL normalization ( $n=3-4 /$ group). Data indicate the mean \pm SEM. ${ }^{*} P<0.05$ versus OLT plus Abx, by Student's $t$ test or 1-way ANOVA followed by Tukey's HSD test.

period (October 2013-August 2015) were analyzed retrospectively, and a total of 264 cases of adult primary OLT were divided into control $(n=108)$ and $\mathrm{Abx}(n=156)$ groups on the basis of equivalent criteria (Figure 7A). As shown in Table 1, patients in the Abx-treated cohort were younger in age $(P=0.001)$, had a higher percentage of alcohol-related liver disease $(P=0.020)$, higher MELD scores $(P<0.001)$, a higher incidence of concomitant HCC $(P<0.001)$, and basiliximab adjunctive therapy $(P<$ 0.001). In parallel, recipients in the Abx treatment group experienced more pre-OLT bloodstream infections $(P<0.001)$, significantly longer preoperative hospital and intensive care unit (ICU) stays, and required larger amounts of blood transfusions during the surgery (Supplemental Table 3). We observed no correlation between Abx grouping and recipient sex, race, height, BMI, disease history, ABO compatibility, serum AST and ALT levels before OLT, or type of Abx used during or after surgery (Table 1). We also found no correlation between Abx grouping and donor/graft parameters, including age, sex, race, height, BMI, pretransplantation blood tests, warm/cold ischemia time, and donor Abx use or donation status (Supplemental Table 4). Remarkably, the Abx treatment plus OLT (Abx-OLT) patient cohort had decreased sALT levels from postoperative day 0 to postoperative day 10 (PODO to POD10) (Figure 7B), suppressed sAST from PODO to POD1 and
POD7 to POD10 (Figure 7B), and a significantly lower incidence of $\operatorname{EAD}(21.8 \%$ vs. $32.4 \%, P=0.0375$; Figure $7 C$ ), despite pretransplantation parameters of more severe clinical acuity (Table 1 ).

To determine whether Abx therapy represents an independent predictor of EAD in OLT, we conducted a multivariate analysis based on a step-wise logistic regression model by screening "pre-OLT Abx-free or Abx $<10$ days" simultaneously with donor Abx use; recipient age, sex, and BMI; MELD score; disease etiology; concomitant HCC; cold/warm ischemia time; blood transfusion amounts; donor age, sex, and BMI; donation after cardiac death (DCD); donor laboratory data (AST, ALT, total bilirubin [T-bil]); Abx treatment during surgery; as well as post-OLT Abx and basiliximab treatment. Of note, "pre-OLT Abx-free or Abx $<10$ days" was identified as one of the predictive factors of EAD ( $\mathrm{OR}=1.970,95 \% \mathrm{CI}: 1.093-3.552, P=0.024)$, along with donor BMI $\left(\mathrm{kg} / \mathrm{m}^{2}, \mathrm{OR}=1.051,95 \%\right.$ CI: $\left.1.001-1.104, P=0.047\right)$ and cold ischemia time (minutes, OR $=1.004,95 \%$ CI: 1.002-1.006, $P<0.001$ ) (Figure 7D). The incidence of post-OLT SSI (ClavienDindo criteria grade $\geq \mathrm{IIIb}$, control $=6.5 \%$ vs. $\mathrm{Abx}=1.3 \%, P=$ 0.317 ) and bloodstream infections (control $=3.7 \%$ vs. $\mathrm{Abx}=7.1 \%$, $P=0.291)$ was comparable between the control and Abx groups (Supplemental Table 5), despite higher pretransplantation liver disease acuity in the latter group (Table 1). 

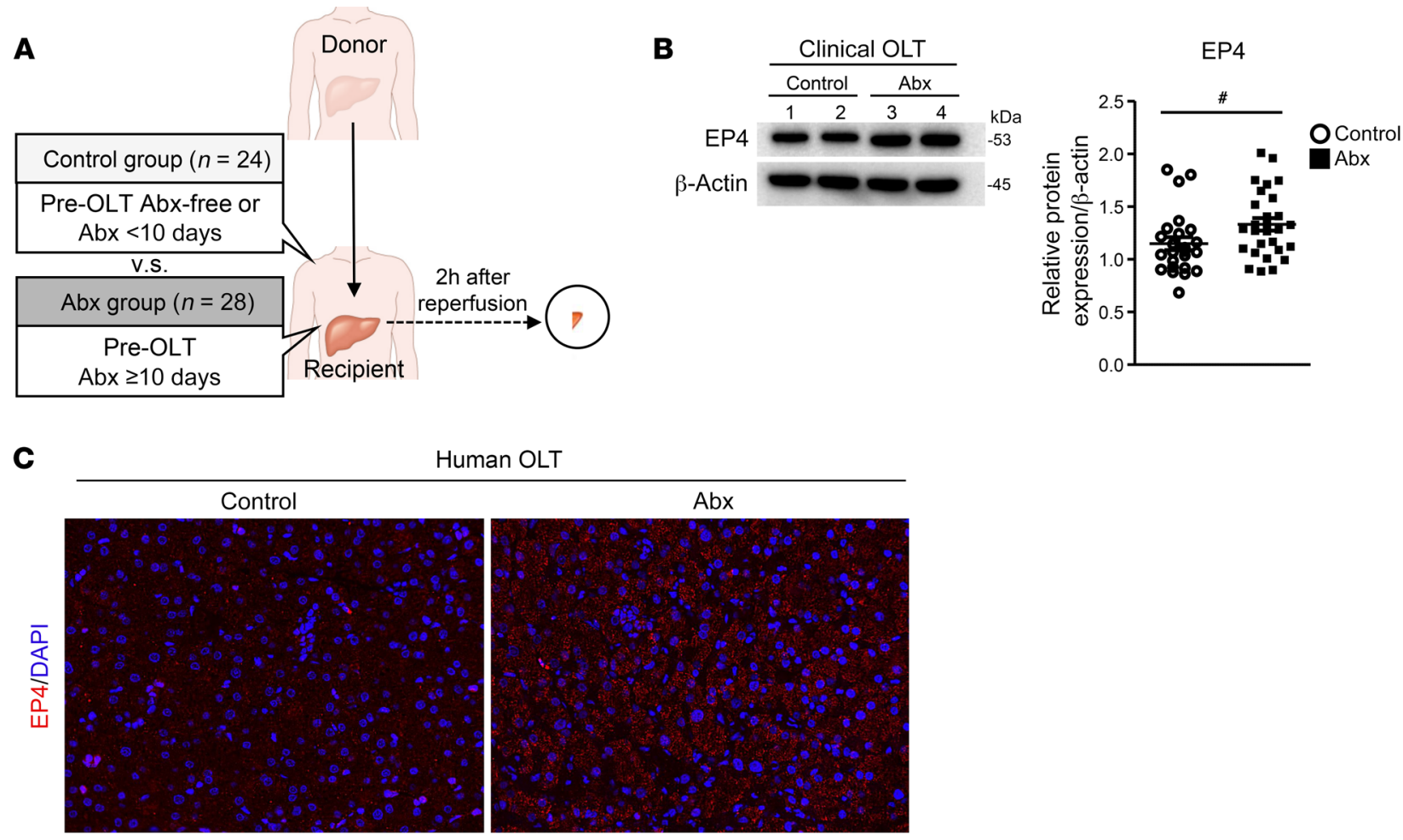

Figure 5. Abx recipient treatment correlates with enhanced hepatic EP4 expression in OLT patients. (A) Protocol liver Bx samples were collected 2 hours after portal reperfusion (prior to the abdominal closure) from 52 primary liver transplantation patients (October 2013-August 2015). OLT recipients were classified into control (recipients with pre-OLT Abx $<10$ days or without Abx; $n=24$ ) and Abx treatment (recipients with pre-OLT Abx $\geq 10$ days; $n=28$ ) groups. (B) Representative Western blot detection of EP4 (cases 1 and 2: control group, cases 3 and 4: Abx group) and Western blot quantification of EP4 with $\beta$-actin normalization. Data indicate the mean \pm SEM. ${ }^{\#} P<0.05$, by Mann-Whitney $U$ test. (C) Representative EP4 staining pattern in human OLT. Original magnification, $\times 200$.

Extended rifaximin pretreatment prevents EAD in human clinical $O L T$. Up to this point, our translational studies have demonstrated benefits of recipient Abx pretreatment in a mouse liver transplant model and in human liver transplant patients. Keeping in mind the diverse clinical use of Abx, we identified rifaximin (RFX), an FDA-approved, minimally absorbed oral antimicrobial agent with broad activity within the gut, as the most frequently used Abx in our clinical cohort (Supplemental Figure 2).

We elected to refine our assessment of the influence of $\mathrm{Abx}$ on clinical outcomes by focusing on selective RFX monotherapy in our patient cohort ( $n=264$; October 2013-August 2015). However, with only 118 subjects remaining after excluding recipients treated with Abx other than RFX prior to OLT $(n=146)$, we extended the observation period and analyzed the impact of pre-OLT Abx in 415 consecutive cases of OLT (January 2013-July 2016). Of these 415 patients, 235 recipients treated with Abx other than RFX prior to OLT were excluded, and the remaining 180 patients were classified into the control (pre-OLT Abx-free or pre-OLT RFX $<10$ days, $n=123$ ) or RFX treatment (recipients with pre-OLT RFX $\geq 10$ days, $n=57$ ) group (Figure $8 \mathrm{~A}$ ). As shown in Table 2, patients in the RFX group were younger in age $(P=0.029)$, had a higher BMI $(P$ $=0.027)$, a higher MELD score $(P<0.001)$, a higher incidence of HCC $(P<0.001)$, and ampicillin-sulbactam use during the surgery $(P<0.001)$. Patients in the RFX group experienced longer preoperative hospital stays and required larger amounts of blood trans- fusions (Supplemental Table 6). There was no correlation between control/RFX grouping and recipient sex, race, height, disease history/etiology, ABO compatibility, sAST/sALT levels before OLT, post-OLT Abx treatment, or post-OLT basiliximab therapy (Table 2). We also found no correlation between Abx grouping and donor/ graft parameters including age, sex, height, BMI, pretransplantation blood tests; warm/cold ischemia time, donor Abx use, or donation status, except for race $(P=0.008)$ (Supplemental Table 7). Notably, RFX-pretreated human recipients had decreased levels of sALT from POD0 to POD10, sAST from POD0 to POD8 and on POD10 (Figure 8B), and a significantly lower incidence of EAD (15.8\% vs. $30.1 \%, P=0.029$; Figure $8 \mathrm{C}$ ), despite pretransplantation parameters of more severe clinical acuity (Table 2).

To determine whether RFX treatment is an independent predictor of EAD in liver transplant patients, we conducted multivariate analysis based on a step-wise logistic regression model by screening "pre-OLT Abx-free or RFX $<10$ days" simultaneously with donor Abx use; recipient age, sex, and BMI; MELD score; disease etiology; concomitant HCC; cold/warm ischemia time; blood transfusions; donor age, sex, BMI, and race; DCD; donor laboratory data (AST, ALT, T-bil); Abx use during surgery; as well as postOLT Abx and basiliximab therapy. Notably, "pre-OLT Abx-free or RFX $<10$ days" was identified as one of the predictive factors of $\mathrm{EAD}(\mathrm{OR}=2.530,95 \% \mathrm{CI}: 1.031-6.210, P=0.043)$, along with donor BMI $\left(\mathrm{kg} / \mathrm{m}^{2}, \mathrm{OR}=1.077,95 \%\right.$ CI: 1.009-1.150, $\left.P=0.026\right)$; 
A

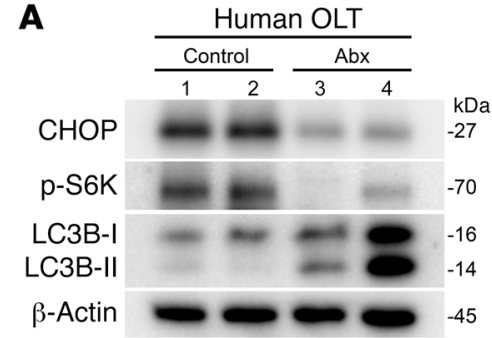

B
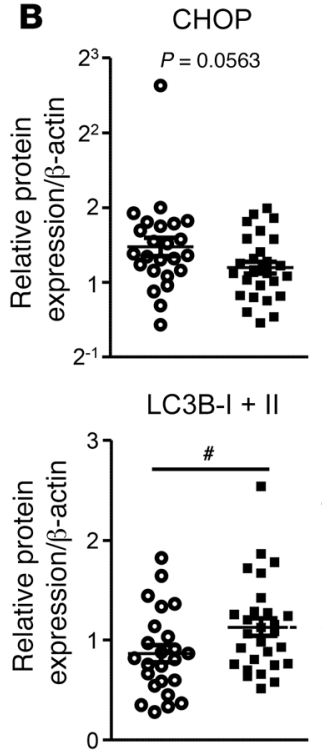

LC3B-II
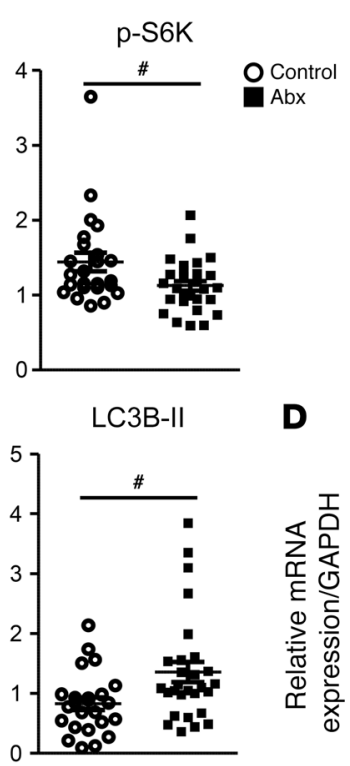

C

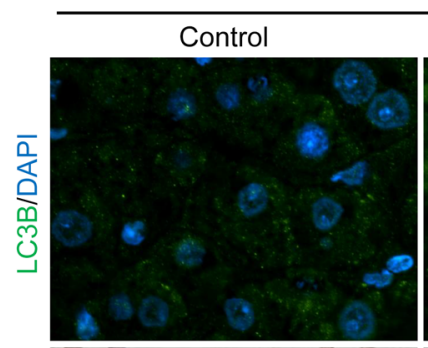

Human OLT
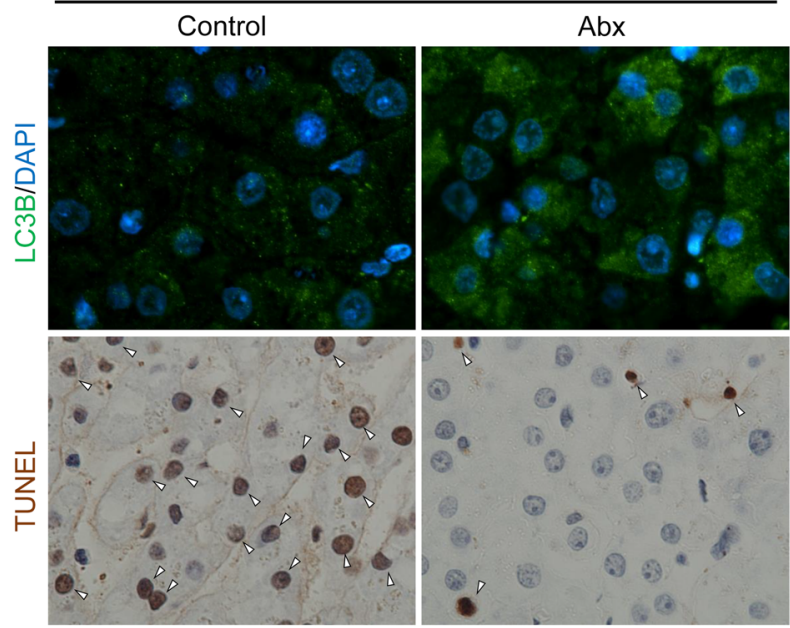

D

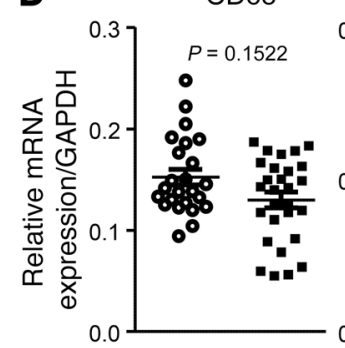

Cathepsin G

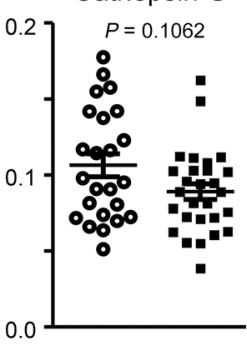

E

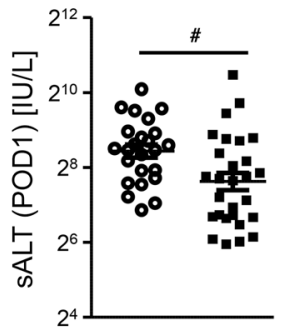

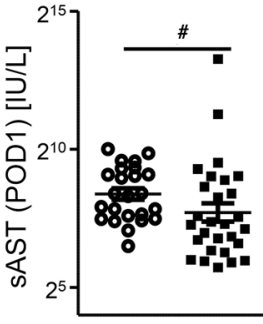

Figure 6. Abx treatment is associated with decreased CHOP and p-S6K, increased LC3B, and lower serum liver enzyme levels (POD1) in OLT recipients. Human OLT Bx samples collected 2 hours after reperfusion $(n=52)$ were classified into control (recipients with pre-OLT Abx $<10$ days or without Abx; $n$ $=24$ ) and Abx treatment (recipients with pre-OLT Abx $\geq 10$ days; $n=28$ ) groups (see also Figure $5 A$ ). (A) Representative Western blot detection of CHOP, p-S6K, and LC3B (cases 1 and 2: control group; cases 3 and 4: Abx group). (B) Western blot quantification of CHOP, p-S6K, LC3B-I plus LC3B-II, and LC3B-II with $\beta$-actin normalization. (C) Representative LC3B and TUNEL staining in human OLT. Arrowheads indicate TUNEL+ dead cells. Original magnification, $\times 400$. (D) qRT-PCR detection of mRNA coding for CD68 and cathepsin G. Data were normalized to GAPDH gene expression. (E) sALT and sAST levels on POD1. Data indicate the mean \pm SEM. ${ }^{P} P<0.05$, by Mann-Whitney $U$ test.

cold ischemia time (minutes, OR $=1.004,95 \% \mathrm{CI}: 1.001-1.007$, $P=0.014)$; donor age (years, $\mathrm{OR}=1.043,95 \% \mathrm{CI}: 1.016-1.071, P=$ $0.002)$; and DCD (OR $=12.697,95 \%$ CI: $2.631-61.262, P=0.002)$ (Figure 8D). The incidences of post-OLT SSI (Clavien-Dindo criteria grade $\geq \mathrm{IIIb}$, control $=4.1 \%$ vs. $\mathrm{Abx}=3.5 \%, P>0.999)$ and post-OLT bloodstream infections (control $=4.9 \%$ vs. $\mathrm{Abx}=1.8 \%$, $P=0.434$ ) were comparable between the control and RFX groups (Supplemental Table 8), despite higher pretransplantation liver disease acuity in the latter group (Table 2).

\section{Discussion}

Recent research is deepening our understanding of microbiota, an ecosystem of bacteria, archaea, protists, fungi, and viruses in the human gut, as not just an idle bystander but an interconnected and active player in human health and disease. Indeed, compositional changes in the intestinal microbiota, such as dysbiosis, mechanistically contribute to hepatic carbohydrate and lipid metabolism and influence the balance between pro- and antiinflammatory effectors, thereby affecting nonalcoholic fatty liver disease (NAFLD) and its progression to nonalcoholic steatohepatitis (NASH) $(40,41)$. As the contribution of the gut microbiome in liver pathophysiology has emerged as an attractive research field, recent studies have documented putative associations between intestinal microbial characteristics and liver graft status in both animals (42) and humans (43). The loss of intestinal microbiota diversity was accompanied by high Child-Pugh and MELD scores, as well as an increased incidence of rejection episodes and bloodstream infections in liver transplant patients. Indeed, the frequencies of Bacteroides, Enterobacteriaceae, Streptococcaceae, and Bifidobacteriaceae were increased, whereas those of Enterococcaceae, Lactobacillaceae, Clostridiaceae, Ruminococcaceae, and Peptostreptococcaceae were decreased in OLT patients experiencing acute rejection (43). On the other hand, the potential of gut microbiota modifications to affect IRI pathology and underlying mechanisms remain largely unknown (44). Here, by encompassing mouse and human OLT, our translational study identifies the therapeutic potential of pretransplantation microbiome alterations to affect the liver transplant fate by mitigating peritransplantation IR stress via the PGE2/EP4 axis.

In the experimental arm, we document that 10-day recipientonly Abx pretreatment conferred IRI protection in a clinically relevant mouse model of liver allotransplantation. This was evidenced 
A

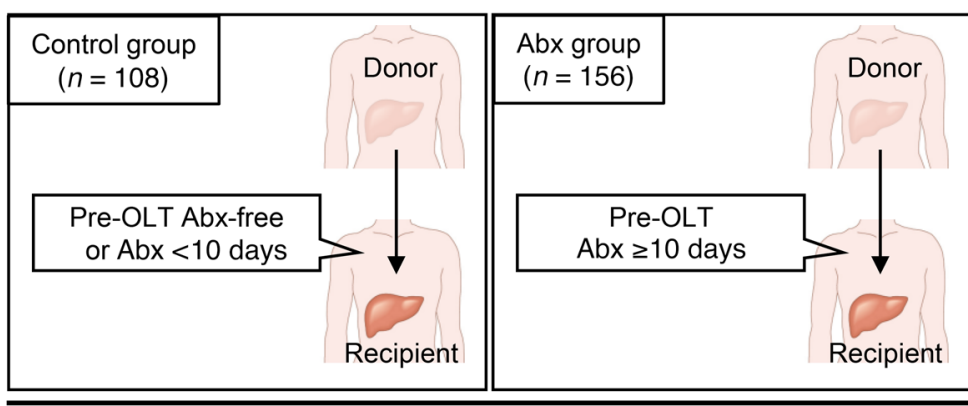

Human OLT between October 2013 and August 2015 ( $n=264$, Table 1/Supp. Table 4)
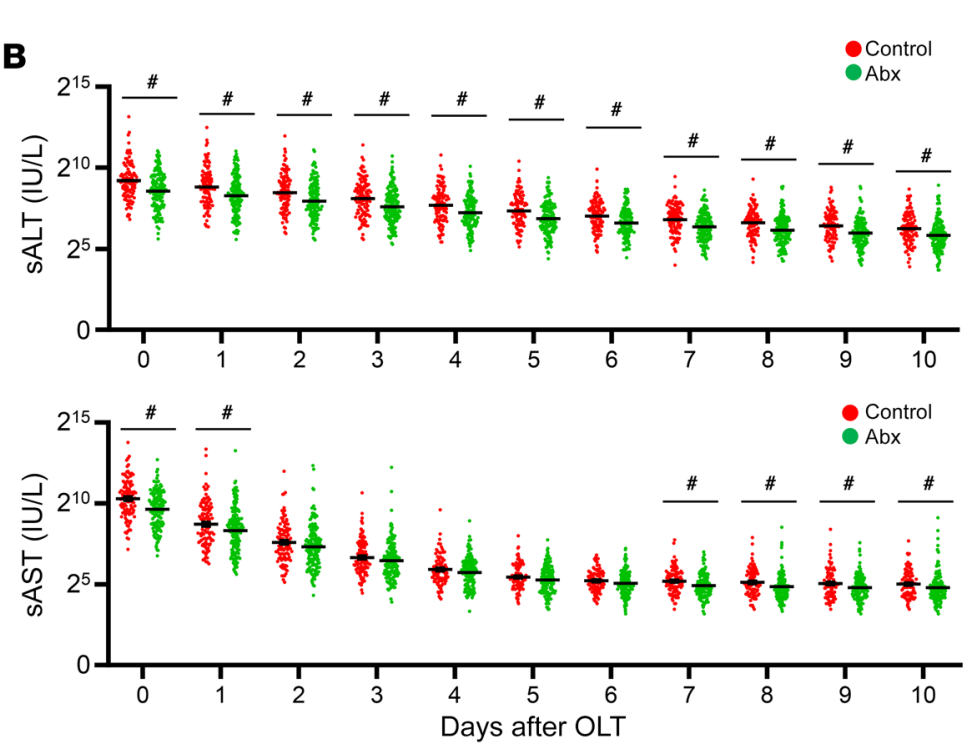
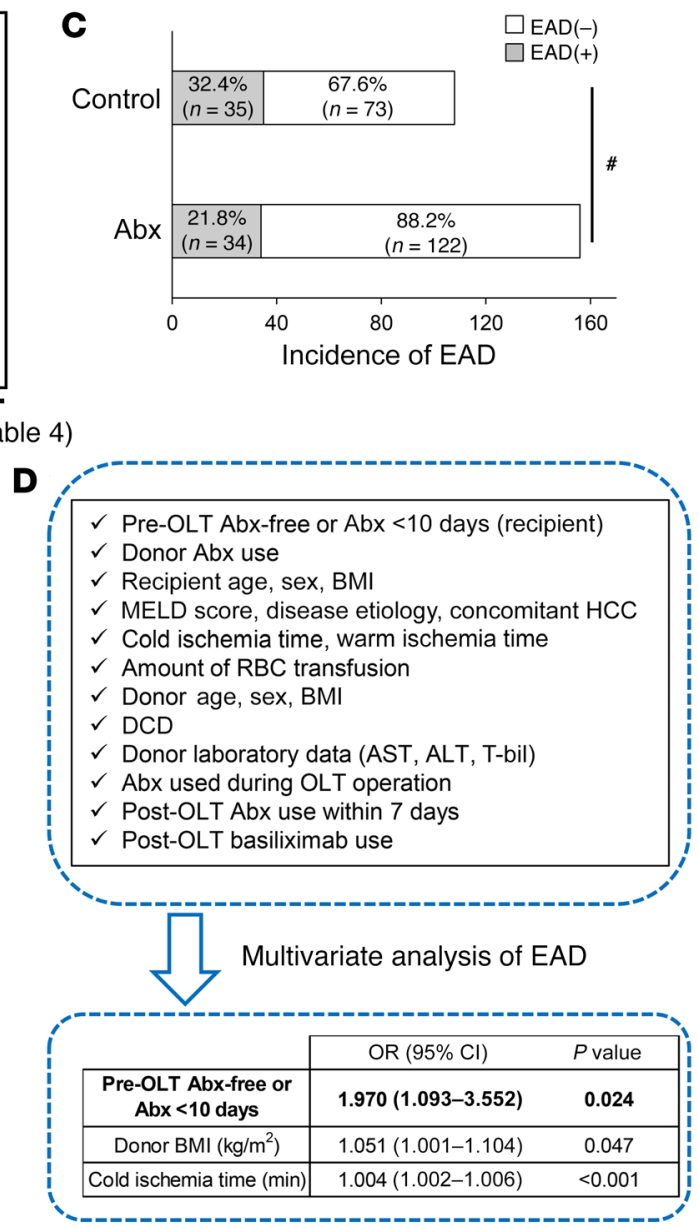

Figure 7. Liver transplant patients with extended Abx treatment ( $\geq 10$ days) experience less EAD. (A) Primary OLT recipients ( $n=264 ; 0 c t o b e r 2013-$ August 2015) were classified into control (pre-OLT Abx $<10$ days or without Abx; $n=108$ ) and Abx treatment (pre-OLT Abx $\geq 10$ days, $n=156$ ) groups. (B) sALT and SAST levels from PODO to POD10. Data indicate the mean \pm SEM. ${ }^{\#} P<0.05$, by Mann-Whitney $U$ test. (C) Incidence of EAD. ${ }^{*} P<0.05$, by Fisher's exact test. (D) Stepwise multivariate logistic regression analysis was performed to identify independent risk factors for EAD.

by suppression of histopathological tissue injury, decreased release of sAST, and modulation of hepatocellular death, accompanied by suppressed HMGB1 release, inhibited leukocyte trafficking, decreased proinflammatory gene programs (Figure 1), as well as suppressed CHOP and increased LC3B expression (Figure 3). Furthermore, gene and protein profiling in our in vitro and in vivo experiments identified the gut-derived PGE2/hepatocyte EP4 signaling axis as a key mechanism in ER stress and autophagy regulation and liver protection (Figures 2-4). Although our study lacks data on the composition of gut microbiota per se, we found, strikingly, that repopulation of Abx-pretreated mouse recipients of FMT from untreated naive donors recreated the cardinal features of hepatic IRI in otherwise stress-resistant OLT, confirming the pathogenic role of the gut microbiome in this model (Supplemental Figure 1).

In the initial clinical arm $(n=52)$, Abx-pretreated human OLT recipients trended toward a phenotype of increased EP4, lower CHOP, higher LC3B, decreased CD68 (macrophage), and suppressed cathepsin G (neutrophil), as well as decreased sALT/sAST levels and a lower incidence of EAD (Figures 5 and 6). Hence, this study highlights previously unappreciated benefits and mechanisms of Abx treatment in preserving hepatocellular function in
IR-stressed OLT. We are aware that some differences between the control and Abx-treated groups failed to reach statistical significance (CHOP, CD68, cathepsin $\mathrm{G}$ ), probably because of the limited number of Bx samples as well as the divergent genetic and environmental backgrounds of human subjects. In our ongoing experiments, Abx treatment decreased $\mathrm{CHOP}$ and enhanced the autophagy pathway in naive mouse livers (our unpublished observations), suggesting that $\mathrm{Abx}$ donor pretreatment may enhance hepatoprotective signaling, consistent with the findings of Corbitt et al., who reported that Abx donor treatment alleviated liver transplant injury (14). However, this strategy is unlikely to be feasible for clinical cadaveric OLT. We observed no significant differences in donor Abx use (Supplemental Tables 4 and 7) and found no association between donor Abx treatment and EAD. Of note, the United Network for Organ Sharing (UNOS) database has its limitations, as unknown timing, duration, and regimen of $\mathrm{Abx}$ use in the donor does not necessarily mean that liver donors were free of adjunctive Abx therapy.

In the extended clinical evaluation of 264 cases, OLT recipients with prolonged ( $\geq 10$ days) Abx pretreatment had a significantly higher pretransplantation "true" MELD score and a lon- 
Table 1. Recipients' perioperative variables (264 cases, October 2013-August 2015)

\begin{tabular}{|c|c|c|c|}
\hline Variables & Control $(n=108)$ & Abx $(n=156)$ & $P$ value \\
\hline Age (yr) & $61(18-75)$ & $56(20-78)$ & 0.001 \\
\hline $\operatorname{Sex}(M / F)$ & $65(60.2 \%) / 43(39.8 \%)$ & $98(62.8 \%) / 58$ (37.2\%) & 0.700 \\
\hline Race & 0.127 & & \\
\hline White & $35(32.4 \%)$ & $64(41.0 \%)$ & \\
\hline Hispanic & $47(43.5 \%)$ & $66(42.3 \%)$ & \\
\hline Black & $8(7.4 \%)$ & $7(4.5 \%)$ & \\
\hline Asian & $15(13.9 \%)$ & $10(6.4 \%)$ & \\
\hline Others & $3(2.8 \%)$ & $9(5.8 \%)$ & \\
\hline Height (cm) & $170.1(142.2-193.0)$ & $167.6(137.2-195.6)$ & 0.680 \\
\hline BMI $\left(\mathrm{kg} / \mathrm{m}^{2}\right)$ & $26.5(16.6-43.3)$ & $26.9(14.5-47.5)$ & 0.368 \\
\hline \multicolumn{4}{|l|}{ History } \\
\hline Smoking & $52(48.1 \%)$ & $75(48.1 \%)$ & $>0.999$ \\
\hline Diabetes & $30(27.8 \%)$ & $54(34.6 \%)$ & 0.283 \\
\hline Hypertension & $50(46.3 \%)$ & $69(44.2 \%)$ & 0.802 \\
\hline Heart disease & $20(18.5 \%)$ & $24(15.4 \%)$ & 0.507 \\
\hline Abdominal surgery & $46(42.6 \%)$ & $60(38.4 \%)$ & 0.525 \\
\hline Disease etiology & & & 0.020 \\
\hline HBV & $5(4.6 \%)$ & $3(1.9 \%)$ & \\
\hline $\mathrm{HCV}$ & $53(49.1 \%)$ & $59(37.8 \%)$ & \\
\hline EtOH & $11(10.2 \%)$ & $38(24.4 \%)$ & \\
\hline Cryptogenic cirrhosis/NASH & $14(13.0 \%)$ & $29(18.6 \%)$ & \\
\hline ALF & $10(9.3 \%)$ & $8(5.1 \%)$ & \\
\hline Others & $15(13.9 \%)$ & $19(12.2 \%)$ & \\
\hline HCC (with/without) & $67(62.0 \%) / 41(38.0 \%)$ & $36(23.1 \%) / 120(76.9 \%)$ & $<0.001$ \\
\hline $\mathrm{ABO}$ & & & 0.352 \\
\hline Identical & $102(94.4 \%)$ & $142(91.0 \%)$ & \\
\hline Compatible & $6(5.6 \%)$ & $14(9.0 \%)$ & \\
\hline MELD score & $14(6-46)$ & $38(7-47)$ & $<0.001$ \\
\hline Pretransplantation AST (IU/L) & $66.5(20-1918)$ & $69(18-5238)$ & 0.309 \\
\hline Pretransplantation ALT (IU/L) & $34.5(12-3705)$ & $35(8-5025)$ & 0.546 \\
\hline Abx use during OLT & & & 0.054 \\
\hline Ampicillin-sulbactam & $75(69.4 \%)$ & $87(55.8 \%)$ & \\
\hline Piperacillin/tazobactam & $13(12.0 \%)$ & $34(21.8 \%)$ & \\
\hline Others & $20(18.5 \%)$ & $35(22.4 \%)$ & \\
\hline Post-OLT Abx use within 7 days & $67(62.0 \%)$ & $112(71.8 \%)$ & 0.109 \\
\hline Post-OLT basiliximab use & $32(29.6 \%)$ & $102(65.4 \%)$ & $<0.001$ \\
\hline
\end{tabular}

ALF, acute liver failure; HBV, hepatitis B virus; HCV, hepatitis C virus; $M$, male; F, female.

demographic variables (age, disease etiology, concomitance of HCC, MELD score, post-OLT basiliximab; Table 1). Indeed, as shown in Figure 7D, we identified "pre-OLT Abx-free or Abx $<10$ days" as one of the independent predictors of EAD (OR $=1.970)$, together with well-established prognostic factors, i.e., donor $\mathrm{BMI}(\mathrm{OR}=1.051)$ and cold ischemia time $(\mathrm{OR}=1.004)$. We acknowledge the limitation of this study, as hepatocellular function was assessed relatively early after transplantation, whereas the evolution of IRI at the cellular, tissue, and immunological levels continues, and the long-term clinical outcomes will require assessment. However, by integrating mouse and human data, our study clearly underscores the benefits of extended recipient Abx pretreatment in OLT recipients.

Since pre-OLT Abx may have influenced decision making regarding the subsequent Abx regimen, we next examined the type of Abx used during and for 7 days after surgery (Table 1). We found no significant differences between the 2 groups, whereas the multivariate analysis excluded those factors from the independent predictors of EAD (Figure 7D), confirming the importance of pre-OLT Abx to prevent EAD. On the other hand, basiliximab was more frequently used in the Abx-OLT patient group, probably because of a concomitant decline in their renal function. However, multivariate analysis failed to identify basiliximab as an independent factor of EAD, indicating that the predictive power of "pre-OLT $A b x \geq 10$ days" to avert EAD was probably not influenced by adjunctive basiliximab therapy.

Unlike in humans, experimental rodents housed in the same facility have relatively equivalent microbiota. However, because of a wide discrepancy between mouse and

ger hospital and ICU stay and required more blood transfusions during the surgery, all associated with the highest-acuity patients compared with controls (Table 1 and Supplemental Table 3). This is not totally unexpected, as Abx are often used to treat concomitant infections or encephalopathy, whereas the occurrence of infection or encephalopathy in a patient on the transplant waiting list increases the MELD score (45-47). However, it is remarkable that despite pretransplantation parameters of severe acuity, the patient cohort with extended Abx pretreatment showed superior postoperative hepatocellular function (Figure 7, B and C), consistent with our mouse OLT data (Figure 1). In addition, considering that our group classification in the human study (control vs. Abx) (Figure 7A) was biased in terms of recipient preoperative variables (Table 1), we conducted a multivariate analysis to estimate the independent influence of pre-OLT Abx on EAD by simultaneously screening putative prognostic factors and the biased recipient human microbiota composition, we acknowledge the need for future integrated studies that will identify the microbiome-Abx interplay, which may be decisive in the OLT outcome. We are also aware that the emergence of drug-resistant bacteria and economic efficiency are discouraging Abx overuse and that evaluation of appropriate Abx regimens (dose, route, length, etc.) and complementary gut microbiome alteration strategies (prebiotics, probiotics, microbiota transplantation, etc.) await future studies.

RFX is a minimally absorbed oral antimicrobial agent that has broad-spectrum activity against both aerobic and anaerobic gram-positive and gram-negative microorganisms within the gut, with a low risk of inducing bacterial resistance (48). First approved in the United States in 2004, RFX is now widely used for the treatment of hepatic encephalopathy (49), improving survival and reducing the risk of hospitalization and portal hypertension (50). Among patients awaiting OLT, those with a history of hepatic encephalopa- 
A

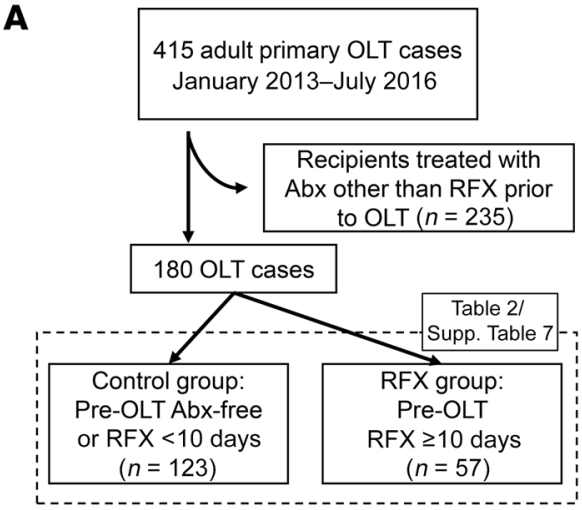

C

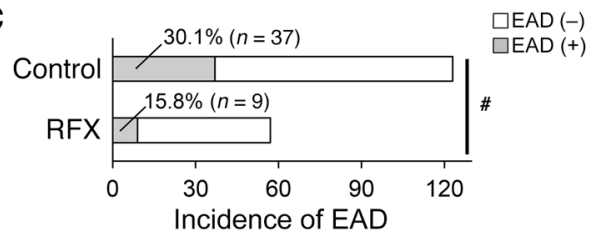

B
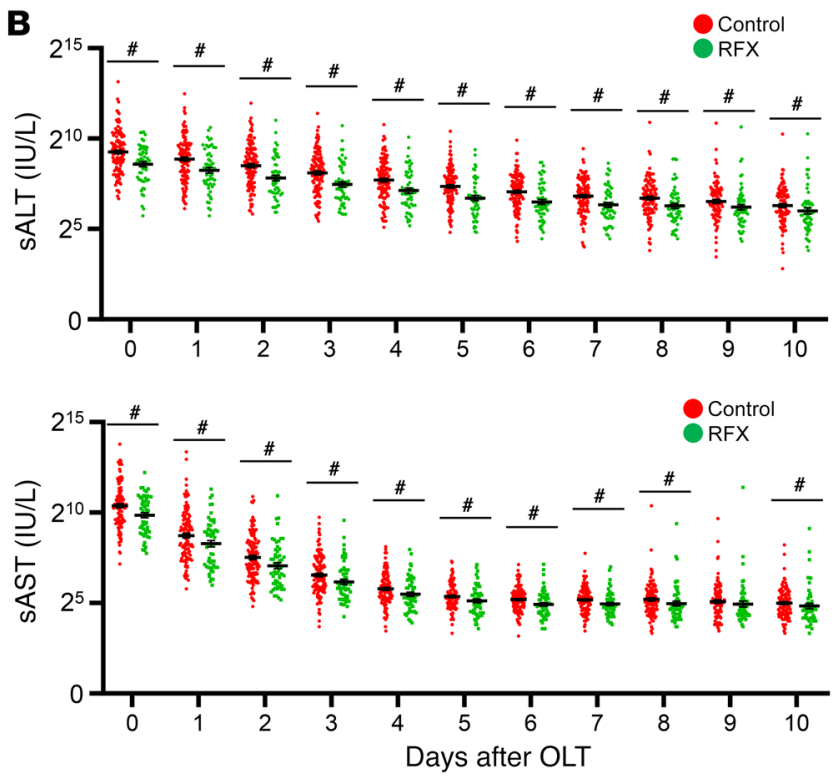

D

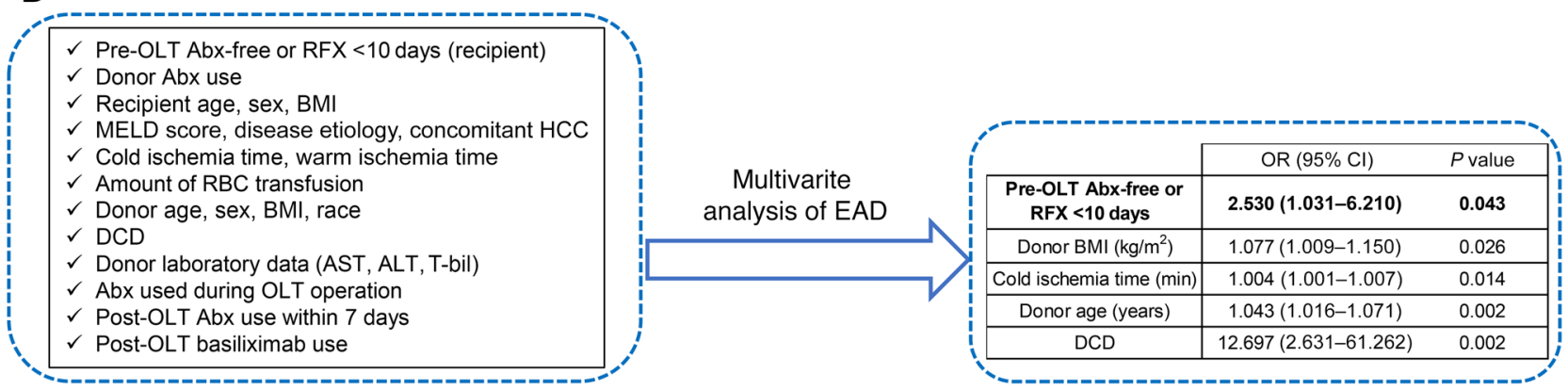

Figure 8. OLT recipients with prolonged RFX therapy ( $\geq 10$ days) experience less EAD. (A) Primary OLT recipients ( $n=415$; January $2013-J u l y ~ 2016)$ were screened for the type of pre-OLT Abx regimen. After 235 recipients treated with Abx other than RFX prior to OLT were excluded, the remaining 180 OLT patients were classified into control (pre-OLT Abx-free or pre-OLT RFX $<10$ days, $n=123$ ) and RFX treatment (recipients with pre-OLT RFX $\geq 10$ days, $n=57$ ) groups. (B) sALT and sAST levels from PODO to POD10. Data indicate the mean \pm SEM. ${ }^{P}<0.05$, by Mann-Whitney $U$ test. (C) Incidence of EAD. $P P<0.05$, by Fisher's exact test. (D) Stepwise multivariate logistic regression analysis was performed to identify independent risk factors of EAD.

thy are treated with RFX, and a protective effect of RFX was reported in post-OLT infection, without increasing multidrug-resistant bacterial infections $(51,52)$. As the principal Abx was applied for prolonged periods in our study (Supplemental Figure 2), we then focused on the influence of RFX monotherapy on OLT clinical outcomes. Indeed, the "pre-OLT RFX $\geq 10$ days" regimen was associated with suppressed post-OLT liver enzyme levels (Figure 8B) and a decreased incidence of EAD (Figure 8C), whereas multivariate analysis identified "pre-OLT RFX $\geq 10$ days" as one of the predictors to avert $\mathrm{EAD}(\mathrm{OR}=2.530)$ (Figure $8 \mathrm{D})$. By excluding recipients treated with Abx other than RFX (Figure 8A), our findings further support the notion that RFX-mediated microbiota modifications may prove clinically beneficial. However, given the conflicting data on the effects of gut Abx regimens in modifying transplantation outcomes, future randomized, controlled clinical trials with larger patient cohorts studied over longer periods are needed to establish the efficacy of RFX in targeting post-OLT IRI and EAD in humans.

Despite the hepatoprotective roles of PGE2 signaling in a mouse warm liver IRI model (33) as well as of its EP4 binding partner expressed primarily by IR-stressed hepatocytes (Fig- ure $2 \mathrm{G}$ and ref. 33), little is known about the hepatocyte PGE2/ EP4 axis. In our current in vitro study, we found that exogenous PGE2 inhibited CHOP and mTORC1, while enhancing the autophagy pathway via EP4 signaling (Figure 3, A-C). In parallel, the EP4 antagonist restored CHOP and p-S6K, decreased LC3B, and recreated IRI in otherwise stress-resistant, Abx-pretreated OLT recipients. Meanwhile, the influence of PGE2 on mTORC1 and cellular autophagy seems to be tissue specific (53-56), whereas the mechanism by which the PGE2/EP4 axis regulates hepatocyte ER stress and mTORC1 activation remains unknown. Consistent with a previous study (57), we found that treatment with PGE2 caused phosphorylation of AMPK in hepatocytes (our unpublished data), while AMPK is known to suppress mTORC1 activation as well as decrease the ER stress response $(58,59)$. Hence, AMPK may be a key molecule regulating hepatocyte ER stress and autophagy. On the other hand, knockdown of CHOP enhanced autophagy in Hepa 1-6 cells (60), whereas CHOP deficiency augmented autophagy in IR-stressed hepatocytes and livers (our unpublished observations). Thus, decreased CHOP signaling under the control of the PGE2/ EP4 axis may enhance autophagic signaling and function. 
Table 2. Recipients' perioperative variables (180 cases in the RFX study, May 2013April 2016)

\begin{tabular}{|c|c|c|c|}
\hline Variables & Control $(n=123)$ & RFX $(n=57)$ & $P$ value \\
\hline Age (yr) & $60(21-76)$ & $59(21-69)$ & 0.029 \\
\hline $\operatorname{Sex}(M / F)$ & $83(67.5 \%) / 40(32.5 \%)$ & $42(73.7 \%) / 15(26.3 \%)$ & 0.254 \\
\hline Race & & & 0.487 \\
\hline White & $43(35.0 \%)$ & $16(28.1 \%)$ & \\
\hline Hispanic & $57(46.3 \%)$ & $28(49.1 \%)$ & \\
\hline Black & $9(7.3 \%)$ & $5(8.8 \%)$ & \\
\hline Asian & $13(10.6 \%)$ & $4(7.0 \%)$ & \\
\hline Others & $1(0.8 \%)$ & $4(7.0 \%)$ & \\
\hline Height (cm) & $170.2(142.2-193.0)$ & $172.7(137.2-195.6)$ & 0.068 \\
\hline BMI $\left(\mathrm{kg} / \mathrm{m}^{2}\right)$ & $26.8(16.1-43.0)$ & $28.4(16.6-47.5)$ & 0.027 \\
\hline \multicolumn{4}{|l|}{ History } \\
\hline Smoking & 56 (54.5\%) & 26 (45.6\%) & $>0.999$ \\
\hline Diabetes & $42(34.1 \%)$ & $17(29.8 \%)$ & 0.612 \\
\hline Hypertension & $57(46.3 \%)$ & $24(42.1 \%)$ & 0.632 \\
\hline Heart disease & $21(17.1 \%)$ & $10(17.5 \%)$ & $>0.999$ \\
\hline Abdominal surgery & $59(48.0 \%)$ & $22(38.6 \%)$ & 0.263 \\
\hline Disease etiology & & & 0.077 \\
\hline HBV & $10(8.1 \%)$ & 0 & \\
\hline HCV & $67(54.5 \%)$ & $29(50.9 \%)$ & \\
\hline $\mathrm{EtOH}$ & $9(7.3 \%)$ & $8(14.0 \%)$ & \\
\hline Cryptogenic cirrhosis/NASH & $14(11.4 \%)$ & $12(21.1 \%)$ & \\
\hline ALF & $6(13.8 \%)$ & $3(5.3 \%)$ & \\
\hline Others & $17(13.8 \%)$ & $5(8.8 \%)$ & \\
\hline HCC (with/without) & $89(72.4 \%)$ & $21(36.8 \%)$ & $<0.001$ \\
\hline $\mathrm{ABO}$ & & & 0.709 \\
\hline Identical & $118(95.9 \%)$ & $54(94.7 \%)$ & \\
\hline Compatible & $5(4.1 \%)$ & $3(5.3 \%)$ & \\
\hline MELD score & $13(6-44)$ & $29(9-47)$ & $<0.001$ \\
\hline Pretransplantation AST (IU/L) & $58(20-1918)$ & $67(20-644)$ & 0.083 \\
\hline Pretransplantation ALT (IU/L) & $32(9-3705)$ & $33(11-297)$ & 0.837 \\
\hline Abx use during OLT & & & $<0.001$ \\
\hline Ampicillin-sulbactam & $66(53.7 \%)$ & $49(86.0 \%)$ & \\
\hline Piperacillin/tazobactam & $37(30.1 \%)$ & $2(3.5 \%)$ & \\
\hline Others & $20(16.3 \%)$ & $6(10.5 \%)$ & \\
\hline Post-OLT Abx use within 7 days & $69(56.1 \%)$ & $32(56.1 \%)$ & $>0.999$ \\
\hline Post-0LT basiliximab use & $33(26.8 \%)$ & $21(36.8 \%)$ & 0.221 \\
\hline
\end{tabular}

Although EP4 antagonism diminished cytoprotective features (inhibition of ER stress, enhancement of autophagy) in our in vitro (hepatocyte cultures) and in vivo (IR-stressed OLT) studies, we acknowledge that the recipient Abx regimen might indirectly protect hepatocytes by inhibiting proinflammatory responses. First, in the pathophysiology of liver IRI, the innate immune system is stimulated by donor-derived DAMPs released by damaged liver cells, as well as by pathogen-associated molecular patterns (PAMPs) from the recipient's gut microbiome. In this context, pretreatment with polymyxin B suppressed portal endotoxin after reperfusion and alleviated IRI in rat OLT (15). Thus, recipient Abx pretreatment might limit hepatic inflammation, at least in part, by suppressing the endotoxin function. However, despite increased endotoxin levels after reperfusion in both animal and human OLT settings $(15,61)$, the efficacy of endotoxin-targeted therapy remains controversial $(62,63)$. Second, since
PGE2 retained the ability to limit inflammatory cell activation $(36,37)$, increased PGE2 expression in our study might have acted to suppress the immune inflammation response. Indeed, given that some EP4positive nonparenchymal cells were found in IR-stressed livers (Figure 2G), further studies will be needed to determine the significance of the PGE2/EP4 axis. Third, with this study, we have started to identify intestinal microbial products capable of affecting hepatic IR stress and its sensors in OLT. The question remains whether hepatic cells are directly targeted by such microbial products or whether they are poised for targeting by shifts in bacterial composition, intestinal bacterial translocation, and/or signals elicited by intermediary cells in the gut/liver axis. We acknowledge the obvious disparity between the clinical OLT setting and the murine OLT model, as the human recipients were severely diseased, whereas the inbred mice were free of pre-OLT stresses. Nevertheless, our bench-to-bedside study provides evidence that recipient Abx pretreatment modulates hepatocyte ER stress and autophagy pathways by PGE2/EP4 signaling, with resultant OLT resistance against IR damage.

The gut microbiome modification by a nonabsorbed Abx (vancomycin) in high-fat diet-fed mice depressed hepatic ER stress markers, including CHOP (23). Likewise, recipient $\mathrm{Abx}$ pretreatment (amoxicillin) modulated EP4/CHOP/p-S6K signaling in our mouse OLT study. These results indicate that putative microbiome alterations, rather than a direct Abx-liver crosstalk, are crucial for hepatic EP4/CHOP/p-S6K platforms. In addition, the gastrointestinal tract is one of the body's organs with abundant expression of COX2 (34), portal blood contains higher amounts of PGE2 as compared with peripheral blood in the steady state (Figure 2C), and EP4 is mainly expressed by parenchymal cells in periportal areas of IR-stressed liver (Figure $2 \mathrm{G}$ and ref. 33), all supporting the notion that the gut might be decisive for systemic PGE2 effects. Indeed, Abx-mediated gut microbiota alterations were shown to increase PGE2 levels not only in peripheral blood but also in bronchoalveolar lavage, resulting in M2 macrophage polarization in the lungs (35). Interestingly, enhanced PGE2 levels after prolonged Abx treatment was accompanied by overgrowth of a colonized fungal Candida species in the gut (without causing systemic candidemia). However, adjunctive antifungal agent administration to Abx-treated mice diminished serum PGE2 levels (35), whereas Candida not only produced PGE2 but also promoted PGE2 production from host cells $(64,65)$. Given that Candi$d a$ infections are not uncommon in OLT (66) and that the role of colonized gastrointestinal fungi in transplantation remains poorly 
understood, future studies on the broad spectrum of the microbiome are warranted.

In conclusion, our translational study documents the hepatoprotective function of $\mathrm{Abx}$ recipient pretreatment and identifies crosstalk between PGE2-EP4 and ER stress and autophagy molecular platforms as the underlying homeostatic defense mechanism against IR stress in mouse and human OLT. This study provides evidence that the gut microbiome not only contributes to IRI pathophysiology but may also represent a potential target for therapeutic intervention to improve liver transplant outcomes.

\section{Methods}

Clinical liver transplant study. Using a database of prospectively collected data, we performed a retrospective analysis of adult patients (age $\geq$ 18 years) who underwent OLT at UCLA, from October 2013 through August $2015(n=264)$. Patients who underwent retransplantation were excluded from the study. Routine pre-OLT prophylactic Abx (neomycin and erythromycin [1 g, 4 times, once per hour], ampicillin-sulbactam [ $3 \mathrm{~g}$, once], on the day before or day of OLT) were administered, as specified by UCLA liver transplant protocols. We routinely use tripleimmunosuppresive therapy (tacrolimus, mycophenolate, and a steroid) according to our institutional protocol, whereas basiliximab is sometimes administrated early after OLT to patients with reduced renal function. The patients were divided on the basis of the duration of their pre-OLT Abx treatment (except for preventive Abx, per the protocol) into the following recipient treatment groups: control (Abx-free or Abx $<10$ days; $n=108$ ) and Abx (Abx $\geq 10$ days; $n=156)$. The MELD score was used to assess the severity of liver disease ("true" MELD score). In the follow-up RFX study, 415 consecutive OLT patients (January 2013July 2016) were screened for the Abx regimen. Recipients treated with Abx other than RFX prior to OLT $(n=235)$ were excluded, while the remaining 180 patients were classified into the control (pre-OLT Abxfree or pre-OLT RFX $<10$ days, $n=123$ ) or RFX (recipients with preOLT RFX $\geq 10$ days, $n=57$ ) treatment group.

Donor livers, procured using standardized techniques after brain or cardiac death, were perfused with and stored in University of Wisconsin (UW) solution (Niaspan, Bristol-Meyers Squibb). Cold ischemia time was defined as the time from perfusion of the donor liver with UW solution to its removal from cold storage for implantation. Warm ischemia time was defined as the time from removal from cold storage to establishment of liver graft reperfusion. Recipients' blood was collected prior to the transplantation procedure and on PODO through POD10 (the POD0 sample was collected at the time of ICU arrival). Hepatocellular function was evaluated by SALT and SAST. EAD was defined by the presence of 1 or more of the following: bilirubin level of $10 \mathrm{mg} / \mathrm{dL}$ or higher on POD7, prothrombin time (PT) and international normalized ratio (INR) of 1.6 or higher on POD7, or sAST and sALT levels above 2000 U/L within the first 7 days. Protocol TruCut needle Bx samples were obtained from liver transplant patients $(n=52)$ approximately 2 hours after portal reperfusion (prior to the abdominal closure) and analyzed by Western blotting and RT-PCR.

Animals. Six- to eight-week-old C57BL/6 and BALB/c mice were used (The Jackson Laboratory). The animals were housed in the UCLA animal facility under specific pathogen-free conditions.

Mouse allogeneic orthotopic liver transplantation. We used a wellestablished mouse model of ex vivo hepatic cold storage followed by OLT, as described previously by our group $(67,68)$. To mimic "mar- ginal" human OLT, donor livers (BALB/c) stored in UW solution at $4^{\circ} \mathrm{C}$ for 18 hours were transplanted into allogeneic (C57BL/6) mice. Recipient mice in the Abx-treated group were conditioned with amoxicillin $(50 \mathrm{mg} / \mathrm{mL}$ in the drinking water; Virbac AH Inc.) for 10 days prior to transplantation $(14,15)$. Some Abx-pretreated OLT recipients were given a selective EP4 antagonist $(5 \mathrm{mg} / \mathrm{kg}$; ONO-AE3-208, R\&D Systems) by forced oral gavage at the time of and 3 hours after reperfusion. Liver graft and serum samples were collected after 6 hours of reperfusion, the peak of hepatocellular damage in this model. The sham group underwent the same procedures except for OLT.

Stool collection and FMT in OLT recipients. Stools collected from untreated naive C57BL/6 mice were weighed and homogenized in PBS (200 mg/mL). For FMT experiments, recipient C57BL/6 mice fed amoxicillin (50 mg/mL in the drinking water) on days -11 to -1 were then conditioned with or without FMT $(200 \mu \mathrm{l}$ at $200 \mathrm{mg} / \mathrm{mL})$ on days -1 and 0 by forced gavage prior to BALB/c liver transplantation surgery, as described previously (11).

Hepatocellular function assay. sAST levels were measured with Infinity AST Liquid Stable Reagent (Thermo Fisher Scientific) and validated with Validate GC3 (Maine Standards Company).

OLT histology and IRI grading. Formalin-fixed, paraffin-embedded liver sections (5- $\mu$ m-thick) were stained with H\&E. The severity of IRI was graded using Suzuki's criteria (69).

TUNEL assay. Cell death in formalin-fixed, paraffin-embedded liver sections $(5 \mu \mathrm{m})$ was detected using an Apop Tag Plus Peroxidase in Situ Apoptosis Kit (MilliporeSigma). Results were scored semiquantitatively by counting the number of positive cells in $10 \mathrm{HPF} /$ section in a blinded manner.

ELISA. Serum concentrations of HMGB1 (MyBioSource), MCP1 (Thermo Fisher Scientific), and PGEM (Cayman Chemical) were measured with an ELISA kit according to the manufacturer's protocol.

Hepatocyte isolation and cultures. Primary mouse hepatocytes, isolated by a 2-stage collagenase perfusion method (21), were incubated with DMSO or PGE2 ( $5 \mu \mathrm{M}, \mathrm{R} \& \mathrm{D}$ Systems) for up to 60 minutes. In some experiments, an EP4 antagonist (20 nM, ONO-AE3-208) was supplemented 15 minutes prior to the PGE2 incubation.

Western blot analysis. Proteins were extracted from tissue and cell samples and their concentration measured using a BCA Protein Assay Kit (Thermo Fisher Scientific). Equal amounts of protein were electrophoresed, blotted, incubated with a primary $\mathrm{Ab}$, a secondary HRP-conjugated Ab, and developed. Primary Abs detecting COX2 (catalog 12282, clone D5H5), mouse CHOP (catalog 5554, clone D46F1), human CHOP (catalog 2895, clone L63F7), p-S6K (catalog 9234, clone Thr389, 108D2), LC3B (catalog 3868, clone D11), vinculin (VCL) (catalog 18799, clone E1E9V), $\beta$-actin (catalog 12620, clone D6A8) (all from Cell Signaling Technology), and EP4 (catalog 248951-AP; Proteintech) were used. To compare target protein expression levels in multiple human OLT samples, densitometric quantification was performed, as reported previously $(70,71)$. Briefly, in a preliminary study, one of the Bx samples expressing all target proteins was chosen and assigned as a control sample. Equal amounts of protein lysate from each sample were applied to each well and gel, and the target band intensity was expressed as the band intensity relative to that of the positive control in the same gel. The target relative protein value was further normalized according to $\beta$-actin intensity.

qRT-PCR analysis. RNA extracted with an RNAse Mini Kit (QIAGEN) was reverse transcribed into cDNA. qRT-PCR was performed 
using QuantStudio 3 (Applied Biosystems). The primer sequences are listed in Supplemental Table 9. Expression of the target gene was normalized to that of the housekeeping HPRT, TBP, or GAPDH gene.

Immunofluorescence. Mouse liver samples were stained with rabbit anti-CD68 Ab (Abcam), rat anti-Ly6G Ab (BD Biosciences), rabbit anti-EP4 Ab, and rabbit anti-LC3B Ab. Mouse intestine samples were stained with rabbit anti-COX2 $\mathrm{Ab}$. Mouse hepatocytes were stained using rabbit anti-LC3B Ab. Human liver samples were stained with rabbit anti-EP4 Ab and rabbit anti-LC3B Ab. Signals were visualized with secondary Alexa Fluor Abs (Donkey Anti-rat IgG [H+L] Highly Cross Adsorbed Secondary Antibody, Alexa Fluor 488, catalog A-21208; Donkey Anti-rabbit IgG [H+L] Highly Cross-Adsorbed Secondary Antibody, Alexa Fluor 488, catalog A-21206; Goat Anti-rabbit IgG [H+L] Cross-Adsorbed Secondary Antibody, Alexa Fluor 555, catalog A-21428; all from Invitrogen, Thermo Fisher Scientific). Hepatic CD $68^{+}{ }^{+} y 6 \mathrm{G}^{+}$cells were scored semiquantitatively by counting cells in $10 \mathrm{HPF} /$ section $(\times 400)$ in a blinded manner.

Statistics. For mouse experiments, comparisons between 2 groups or multiple groups were assessed using a 2-tailed Student's $t$ test or a 1-way ANOVA followed by Tukey's honestly significant difference (HSD) test, respectively. For human data, continuous values were analyzed by Mann-Whitney $U$ test and categorical variables by Fisher's exact test. Multivariate analysis based on a step-wise logistic regression model was used to identify independent predictive factors of EAD. $P$ values of less than 0.05 were considered statistically significant. JMP for Windows 8.0 (SAS Institute) was used for statistical analyses.

Study approval. All human studies were approved by the IRB of UCLA (protocols 13-000143 and 18-000216), and written informed consent was received from all participants prior to their inclusion in the study. All mouse experiments were approved by the UCLA Animal Research
Committee (protocol 1999-094), and all animals received humane care according to the criteria outlined in the NIH's Guide for the Care and Use of Laboratory Animals (National Academies Press, revised 1985).

\section{Author contributions}

KN, SK, TI, and JWKW were responsible for the study concept and design. KN, SK, HH, KK, and KJD were responsible for experimental data acquisition. SK and $\mathrm{HH}$ performed surgical procedures. $\mathrm{KN}, \mathrm{TI}, \mathrm{AA}, \mathrm{FMK}$ conducted clinical data analyses. MJE, KT, SU, DGF, FMK, and RWB discussed and reviewed the manuscript. KN, SK, TI, and JWKW drafted the manuscript. RWB and JWKW obtained funding. All authors read and edited the manuscript.

\section{Acknowledgments}

We thank Ko Takanashi (Translational Pathology Core Laboratory, UCLA) and Damla Oncel (UCLA) for immunohistochemical staining assistance and Justine Aziz and Stephanie Younan (both from UCLA) for help with clinical data collection. This work was supported by NIH grants P01 AI120944, R01 DK062357, DK107533, and DK102110 (to JWKW); the Terasaki Family Foundation; and the Dumont-UCLA Research Foundation.

Address correspondence to: Jerzy W. Kupiec-Weglinski, DumontUCLA Transplant Center, 77-120 CHS, 10833 Le Conte Avenue, Los Angeles, California 90095, USA. Phone: 310.825.4196; Email: jkupiec@mednet.ucla.edu.

KN's present address is: Department of Surgery, Kyoto University, Kyoto, Japan; and Department of Surgery, Nishi-Kobe Medical Center, Nishi-ku, Kobe, Japan.
1. Dutkowski P, Linecker M, DeOliveira ML, Müllhaupt B, Clavien PA. Challenges to liver transplantation and strategies to improve outcomes. Gastroenterology. 2015;148(2):307-323.

2. Zhai Y, Petrowsky H, Hong JC, Busuttil RW, Kupiec-Weglinski JW. Ischaemia-reperfusion injury in liver transplantation--from bench to bedside. Nat Rev Gastroenterol Hepatol. 2013;10(2):79-89.

3. Ley RE, Peterson DA, Gordon JI. Ecological and evolutionary forces shaping microbial diversity in the human intestine. Cell. 2006;124(4):837-848.

4. Kostic AD, Xavier RJ, Gevers D. The microbiome in inflammatory bowel disease: current status and the future ahead. Gastroenterology. 2014;146(6):1489-1499.

5. Tang WH, Hazen SL. The contributory role of gut microbiota in cardiovascular disease. J Clin Invest. 2014;124(10):4204-4211.

6. Cani PD, et al. Changes in gut microbiota control inflammation in obese mice through a mechanism involving GLP-2-driven improvement of gut permeability. Gut. 2009;58(8):1091-1103.

7. Cani PD, et al. Changes in gut microbiota control metabolic endotoxemia-induced inflammation in high-fat diet-induced obesity and diabetes in mice. Diabetes. 2008;57(6):1470-1481.

8. Sampson TR, et al. Gut microbiota regulate motor deficits and neuroinflammation in a model of Parkinson's disease. Cell. 2016;167(6):1469-1480.e12.

9. Jang HR, Gandolfo MT, Ko GJ, Satpute S, Racu- sen L, Rabb H. Early exposure to germs modifies kidney damage and inflammation after experimental ischemia-reperfusion injury. Am J Physiol Renal Physiol. 2009;297(5):F1457-F1465.

10. Lei YM, et al. The composition of the microbiota modulates allograft rejection. JClin Invest. 2016;126(7):2736-2744.

11. Bromberg JS, et al. Gut microbiota-dependent modulation of innate immunity and lymph node remodeling affects cardiac allograft outcomes. JCI Insight. 2018;3(19):e121045.

12. Upadhyay V, Fu YX, Bromberg JS. From infection to colonization: the role of microbiota in transplantation. Am J Transplant. 2013;13(4):829.

13. McIntosh CM, Chen L, Shaiber A, Eren AM, Alegre ML. Gut microbes contribute to variation in solid organ transplant outcomes in mice. Microbiome. 2018;6(1):96.

14. Corbitt N, et al. Gut bacteria drive Kupffer cell expansion via MAMP-mediated ICAM-1 induction on sinusoidal endothelium and influence preservation-reperfusion injury after orthotopic liver transplantation. Am J Pathol. 2013;182(1):180-191.

15. Arai M, Mochida S, Ohno A, Arai S, Fujiwara K. Selective bowel decontamination of recipients for prevention against liver injury following orthotopic liver transplantation: evaluation with rat models. Hepatology. 1998;27(1):123-127.

16. Dara L, Ji C, Kaplowitz N. The contribution of endoplasmic reticulum stress to liver diseases. Hepatology. 2011;53(5):1752-1763.

17. Liu J, et al. Endoplasmic reticulum stress modulates liver inflammatory immune response in the pathogenesis of liver ischemia and reperfusion injury. Transplantation. 2012;94(3):211-217.

18. Zhou H, et al. The dichotomy of endoplasmic reticulum stress response in liver ischemia-reperfusion injury. Transplantation. 2016;100(2):365-372.

19. Toriguchi K, et al. Attenuation of steatohepatitis, fibrosis, and carcinogenesis in mice fed a methionine-choline deficient diet by CCAAT/enhancer-binding protein homologous protein deficiency. J Gastroenterol Hepatol. 2014;29(5):1109-1118.

20. Lu M, et al. Opposing unfolded-protein-response signals converge on death receptor 5 to control apoptosis. Science. 2014;345(6192):98-101.

21. Tamaki N, et al. CHOP deficiency attenuates cholestasis-induced liver fibrosis by reduction of hepatocyte injury. Am J Physiol Gastrointest Liver Physiol. 2008;294(2):G498-G505.

22. Wada S, et al. CAAT/enhancer binding protein-homologous protein deficiency attenuates liver ischemia/reperfusion injury in mice. Liver Transpl. 2018;24(5):645-654.

23. Fujisaka S, et al. Antibiotic effects on gut microbiota and metabolism are host dependent. J Clin Invest. 2016;126(12):4430-4443.

24. Baehrecke EH. Autophagy: dual roles in life and 
death? Nat Rev Mol Cell Biol. 2005;6(6):505-510.

25. Czaja MJ, et al. Functions of autophagy in normal and diseased liver. Autophagy. 2013;9(8):1131-1158.

26. Kim JS, et al. Impaired autophagy: A mechanism of mitochondrial dysfunction in anoxic rat hepatocytes. Hepatology. 2008;47(5):1725-1736.

27. Wang JH, et al. Autophagy suppresses age-dependent ischemia and reperfusion injury in livers of mice. Gastroenterology. 2011;141(6):2188-2199.e6.

28. Zhu J, et al. Rapamycin protection of livers from ischemia and reperfusion injury is dependent on both autophagy induction and mammalian target of rapamycin complex 2-Akt activation. Transplantation. 2015;99(1):48-55.

29. Nakamura K, et al. Heme oxygenase-1 regulates sirtuin-1-autophagy pathway in liver transplantation: From mouse to human. Am J Transplant. 2018;18(5):1110-1121.

30. Narumiya S, Sugimoto Y, Ushikubi F. Prostanoid receptors: structures, properties, and functions. Physiol Rev. 1999;79(4):1193-1226.

31. Stachura J, et al. Prostaglandin protection of carbon tetrachloride-induced liver cell necrosis in the rat. Gastroenterology. 1981;81(2):211-217.

32. Takano M, et al. Prostaglandin E2 protects against liver injury after Escherichia coli infection but hampers the resolution of the infection in mice. JImmunol. 1998;161(6):3019-3025.

33. Kuzumoto $Y$, et al. Significance and therapeutic potential of prostaglandin $\mathrm{E} 2$ receptor in hepatic ischemia/reperfusion injury in mice. Hepatology. 2005;42(3):608-617.

34. Kirkby NS, et al. Systematic study of constitutive cyclooxygenase-2 expression: Role of NF- $\mathrm{kB}$ and NFAT transcriptional pathways. Proc Natl Acad Sci U S A. 2016;113(2):434-439.

35. Kim YG, Udayanga KG, Totsuka N, Weinberg JB, Núñez G, Shibuya A. Gut dysbiosis promotes M2 macrophage polarization and allergic airway inflammation via fungi-induced $\mathrm{PGE}_{2}$. Cell Host Microbe. 2014;15(1):95-102.

36. MacKenzie KF, et al. PGE(2) induces macrophage IL-10 production and a regulatory-like phenotype via a protein kinase A-SIK-CRTC3 pathway. JImmunol. 2013;190(2):565-577.

37. Hangai $S$, et al. PGE2 induced in and released by dying cells functions as an inhibitory DAMP. Proc Natl Acad Sci U S A. 2016;113(14):3844-3849.

38. Rabanal-Ruiz Y, Otten EG, Korolchuk VI. mTORC1 as the main gateway to autophagy. Essays Biochem. 2017;61(6):565-584.

39. Yang L, et al. The mTORC1 effectors S6K1 and $4 \mathrm{E}-\mathrm{BP}$ play different roles in CNS axon regeneration. Nat Commun. 2014;5:5416.

40. Brandl K, Schnabl B. Intestinal microbiota and nonalcoholic steatohepatitis. Curr Opin Gastroenterol. 2017;33(3):128-133.

41. Kolodziejczyk AA, Zheng D, Shibolet O, Elinav E. The role of the microbiome in NAFLD and NASH. EMBO Mol Med. 2019;11(2):e9302.
42. Ren Z, et al. Intestinal microbial variation may predict early acute rejection after liver transplantation in rats. Transplantation. 2014;98(8):844-852.

43. Kato K, et al. Longitudinal analysis of the intestinal microbiota in liver transplantation. Transplant Direct. 2017;3(4):e144.

44. Kriss M, Verna EC, Rosen HR, Lozupone CA. Functional microbiomics in liver transplantation: identifying novel targets for improving allograft outcomes. Transplantation. 2019;103(4):668-678

45. Bertuzzo VR, et al. Impact of preoperative infection on outcome after liver transplantation. $\mathrm{Br} \mathrm{J}$ Surg. 2017;104(2):e172-e181.

46. Sun HY, Cacciarelli TV, Singh N. Impact of pretransplant infections on clinical outcomes of liver transplant recipients. Liver Transpl. 2010;16(2):222-228.

47. Wong RJ, Aguilar M, Gish RG, Cheung R, Ahmed A. The impact of pretransplant hepatic encephalopathy on survival following liver transplantation. Liver Transpl. 2015;21(7):873-880.

48. Debbia EA, Maioli E, Roveta S, Marchese A. Effects of rifaximin on bacterial virulence mechanisms at supra- and sub-inhibitory concentrations. JChemother. 2008;20(2):186-194.

49. Scarpignato C, Pelosini I. Experimental and clinical pharmacology of rifaximin, a gastrointestinal selective antibiotic. Digestion. 2006;73(Suppl 1):13-27.

50. Bass NM, et al. Rifaximin treatment in hepatic encephalopathy. $N$ Engl JMed. 2010;362(12):1071-1081.

51. Sun HY, Wagener M, Cacciarelli TV, Singh N. Impact of rifaximin use for hepatic encephalopathy on the risk of early post-transplant infections in liver transplant recipients. Clin Transplant. 2012;26(6):849-852.

52. Esfeh JM, et al. Impact of pretransplant rifaximin therapy on early post-liver transplant infections. Liver Transpl. 2014;20(5):544-551.

53. Domingo-Gonzalez R, et al. Inhibition of neutrophil extracellular trap formation after stem cell transplant by prostaglandin E2. Am J Respir Crit Care Med. 2016;193(2):186-197.

54. Martínez-Colón GJ, Taylor QM, Wilke CA, Podsiad AB, Moore BB. Elevated prostaglandin E. Mucosal Immunol. 2018;11(2):319-332.

55. Chang HH, et al. Prostaglandin E2 activates the mTORC1 pathway through an EP4/cAMP/ PKA- and EP1/Ca2+-mediated mechanism in the human pancreatic carcinoma cell line PANC-1. Am JPhysiol, Cell Physiol. 2015;309(10):C639-C649.

56. Okunishi K, DeGraaf AJ, Zasłona Z, PetersGolden M. Inhibition of protein translation as a novel mechanism for prostaglandin E2 regulation of cell functions. FASEB J. 2014;28(1):56-66.

57. Kainuma S, et al. Regulation by AMP-activated protein kinase of PGE2-induced osteoprotegerin synthesis in osteoblasts. Mol Med Rep. 2016;13(4):3363-3369.

58. Terai K, et al. AMP-activated protein kinase protects cardiomyocytes against hypoxic injury through attenuation of endoplasmic reticulum stress. Mol Cell Biol. 2005;25(21):9554-9575.

59. Liu JQ, Zhang L, Yao J, Yao S, Yuan T. AMPK alleviates endoplasmic reticulum stress by inducing the ER-chaperone ORP150 via FOXO1 to protect human bronchial cells from apoptosis. Biochem Biophys Res Commun. 2018;497(2):564-570.

60. Lei Y, et al. CHOP favors endoplasmic reticulum stress-induced apoptosis in hepatocellular carcinoma cells via inhibition of autophagy. PLoS One. 2017;12(8):e0183680.

61. Yokoyama I, Todo S, Miyata T, Selby R, Tzakis AG, Starzl TE. Endotoxemia and human liver transplantation. Transplant Proc. 1989;21(5):3833-3841.

62. Fiorini RN, et al. Anti-endotoxin monoclonal antibodies are protective against hepatic ischemia/reperfusion injury in steatotic mice. $\mathrm{Am} \mathrm{J}$ Transplant. 2004;4(10):1567-1573.

63. Ziegler EJ, et al. Treatment of gram-negative bacteremia and septic shock with HA-1A human monoclonal antibody against endotoxin. A randomized, double-blind, placebo-controlled trial. The HA-1A Sepsis Study Group. N Engl J Med. 1991;324(7):429-436.

64. Noverr MC, Toews GB, Huffnagle GB. Production of prostaglandins and leukotrienes by pathogenic fungi. Infect Immun. 2002;70(1):400-402.

65. Lee HS, Lee CS, Yang CJ, Su SL, Salter DM. Candida albicans induces cyclo-oxygenase 2 expression and prostaglandin $\mathrm{E} 2$ production in synovial fibroblasts through an extracellular-regulated kinase 1/2 dependent pathway. Arthritis Res Ther. 2009;11(2):R48.

66. Hogen R, Dhanireddy KK. Invasive fungal infections following liver transplantation. Curr Opin Organ Transplant. 2017;22(4):356-363.

67. Kageyama S, et al. Recombinant relaxin protects liver transplants from ischemia damage by hepatocyte glucocorticoid receptor: from benchto-bedside. Hepatology. 2018;68(1):258-273.

68. Kageyama S, Nakamura K, Ke B, Busuttil RW, Kupiec-Weglinski JW. Serelaxin induces Notch1 signaling and alleviates hepatocellular damage in orthotopic liver transplantation. Am J Transplant. 2018;18(7):1755-1763

69. Suzuki S, Toledo-Pereyra LH, Rodriguez FJ, Cejalvo D. Neutrophil infiltration as an important factor in liver ischemia and reperfusion injury. Modulating effects of FK506 and cyclosporine. Transplantation. 1993;55(6):1265-1272.

70. Nakamura K, et al. Sirtuin 1 attenuates inflammation and hepatocellular damage in liver transplant ischemia/reperfusion: from mouse to human. Liver Transpl. 2017;23(10):1282-1293.

71. Nakamura K, et al. Macrophage heme oxygenase1-SIRT1-p53 axis regulates sterile inflammation in liver ischemia-reperfusion injury. J Hepatol. 2017;67(6):1232-1242. 